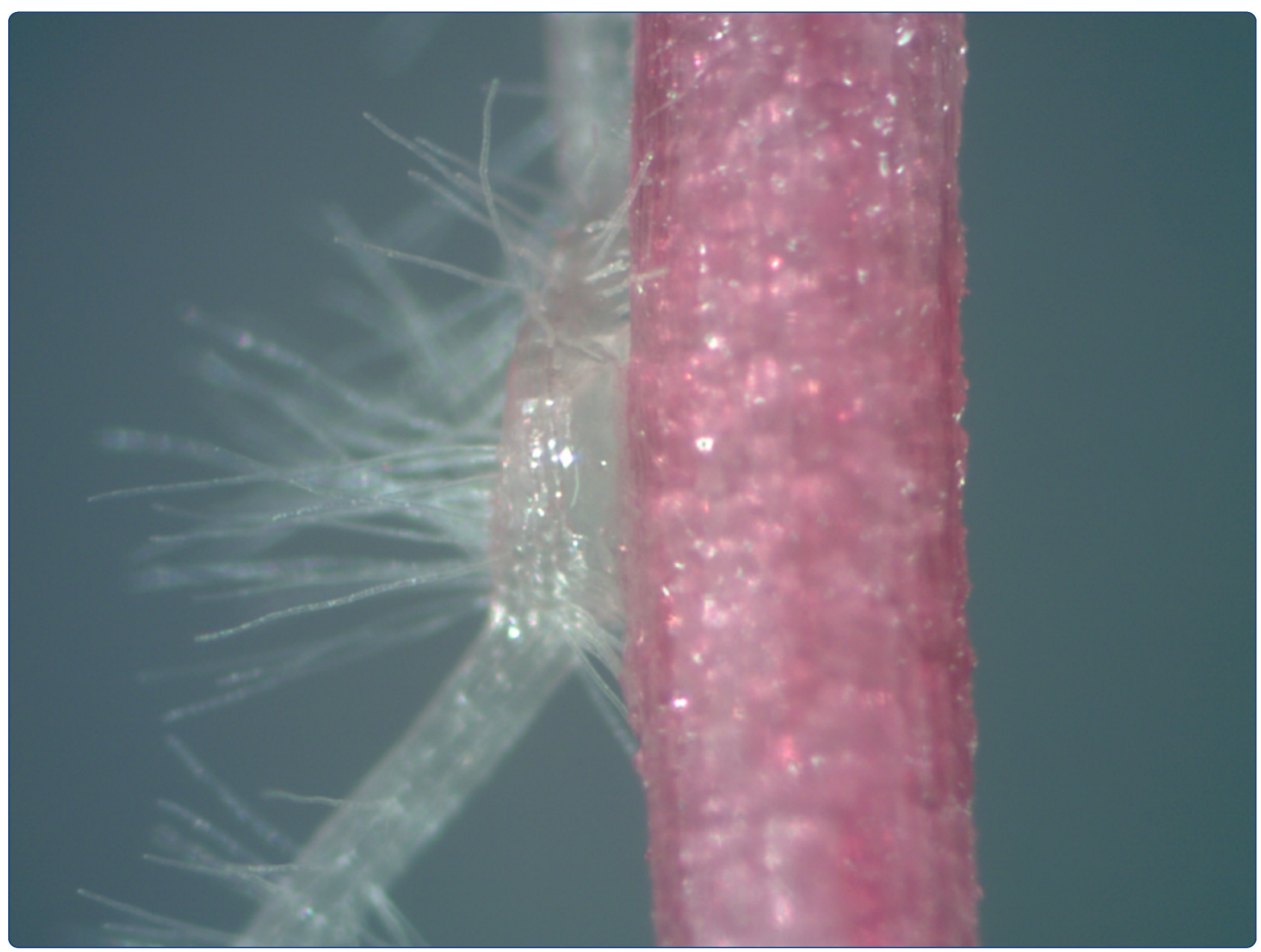

Functional genomics of a generalist parasitic plant: Laser microdissection of host-parasite interface reveals host-specific patterns of parasite gene expression

Honaas et al. 


\title{
Functional genomics of a generalist parasitic plant: Laser microdissection of host-parasite interface reveals host-specific patterns of parasite gene expression
}

Loren A Honaas', Eric K Wafula ${ }^{2}$, Zhenzhen Yang ${ }^{1}$, Joshua P Der ${ }^{1,2}$, Norman J Wickett ${ }^{1,2,8}$, Naomi S Altman ${ }^{3}$, Christopher G Taylor ${ }^{4}$, John I Yoder ${ }^{5}$, Michael P Timko ${ }^{6}$, James H Westwood ${ }^{7}$ and Claude W dePamphilis ${ }^{1,2^{*}}$

\begin{abstract}
Background: Orobanchaceae is the only plant family with members representing the full range of parasitic lifestyles plus a free-living lineage sister to all parasitic lineages, Lindenbergia. A generalist member of this family, and an important parasitic plant model, Triphysaria versicolor regularly feeds upon a wide range of host plants. Here, we compare de novo assembled transcriptomes generated from laser micro-dissected tissues at the host-parasite interface to uncover details of the largely uncharacterized interaction between parasitic plants and their hosts.

Results: The interaction of Triphysaria with the distantly related hosts Zea mays and Medicago truncatula reveals dramatic host-specific gene expression patterns. Relative to above ground tissues, gene families are disproportionally represented at the interface including enrichment for transcription factors and genes of unknown function. Quantitative Real-Time PCR of a T. versicolor $\beta$-expansin shows strong differential (120x) upregulation in response to the monocot host $Z$. mays; a result that is concordant with our read count estimates.

Pathogenesis-related proteins, other cell wall modifying enzymes, and orthologs of genes with unknown function (annotated as such in sequenced plant genomes) are among the parasite genes highly expressed by T. versicolor at the parasite-host interface.

Conclusions: Laser capture microdissection makes it possible to sample the small region of cells at the epicenter of parasite host interactions. The results of our analysis suggest that T. versicolor's generalist strategy involves a reliance on overlapping but distinct gene sets, depending upon the host plant it is parasitizing. The massive upregulation of a T. versicolor $\beta$-expansin is suggestive of a mechanism for parasite success on grass hosts. In this preliminary study of the interface transcriptomes, we have shown that $T$. versicolor, and the Orobanchaceae in general, provide excellent opportunities for the characterization of plant genes with unknown functions.
\end{abstract}

Keywords: Parasitic plant, RNA-Seq, Illumina, De novo assembly, Transcriptome, Laser microdissection, Expansin, Generalist parasite, Orobanchaceae, Triphysaria, Maize, Medicago, Burkholderia

\footnotetext{
* Correspondence: cwd3@psu.edu

${ }^{1}$ Intercollege Graduate Program in Plant Biology, Huck Institutes of the Life Sciences, The Pennsylvania State University, University Park, Pennsylvania 16802, USA

2Department of Biology and Institute of Molecular Evolutionary Genetics, The Pennsylvania State University, University Park, Pennsylvania 16802, USA Full list of author information is available at the end of the article
} 


\section{Background}

Approximately $1 \%$ of all extant angiosperm species are parasitic, deriving all or part of the water and nutrients from host plant species using specialized feeding structures known as haustoria. Among families containing parasites, only the Orobanchaceae contain species representing the full spectrum of parasitism from potentially freeliving facultative forms to non-photosynthetic, obligate parasites [1]. Lindenbergia is a non-parasitic lineage of Orobanchaceae sister to all parasitic species [2]; therefore the family represents an ideal comparative framework to study the evolution of parasitism. Parasitic Orobanchaceae growing in Africa and the Mediterranean include the devastating agricultural pests witchweed (Striga) and broomrape (Orobanche and Phelipanche), respectively. The Striga infestation covers 123.5 million acres resulting in annual yield losses greater than US\$7 billion [3,4]. Broomrapes threaten nearly 40 million acres, though yield losses are difficult to assess due to the frequent abandonment of infested fields and unreliable data on yield loss [5]. Striga is one of the primary biotic constraints to agriculture in Sub-Saharan Africa and the affected areas are increasing in size [6]. The weedy, parasitic Orobanchaceae also threaten parts of Asia, Europe, and North America [7].

Motivated by the agronomic threat presented by some parasitic Orobanchaceae, Triphysaria versicolor has been developed as a model parasitic plant for the family. As a transformable [8] and tractable facultative generalist parasite, $T$. versicolor represents an excellent species to investigate the evolution of parasitism, haustorium development, plant-plant communication, host-parasite interactions, and many other facets of parasite biology $[9,10]$. To discover processes important in parasitic plant biology, we focused our analysis on the unifying anatomical feature of parasitic plants, the haustorium. This modified root structure is adapted to enable feeding on the host and is unique to parasitic plants, thus it is a focal point for interactions between the parasite and host [11].

Heide-Jorgensen and Kuijt [12,13] showed that the haustorium of $T$. versicolor contains many specialized cells including haustorial hairs, a xylem bridge between the host and parasite, and transfer-like cells adjacent to vessel elements at the host-parasite interface. Although histological evidence for xylem connectivity between the haustorium of $T$. versicolor and its host is well documented $[12,13]$, there is no evidence for phloem connectivity. However, there is evidence that phloem-mobile virus particles move between host and parasite in the holoparasite Phelipanche (syn. Orobanche) ramosa [14] and phloem continuity has been observed in Orobanche crenata [15]. The mechanisms of transport between host phloem and parasite phloem likely vary in different parasites from direct phloem connections [15] to transport via apoplastic pathways. Bi-directional movement of small RNAs between host and parasite has been documented in T. versicolor attacking transgenic lettuce [16]. The anatomy of the haustorial interface cells and empirical evidence for bi-directional transport point to the host-parasite interface as an epicenter of host-parasite dialogue.

Intimate symbioses tend towards specialization (e.g. parasitism) [17]. A true generalist strategy, where a parasite routinely feeds on many distantly related host species, is relatively uncommon in parasitic organisms [18]. At face value, this is surprising, because a broad host range provides more feeding opportunities. Seedlings of most parasitic plants, for example, must contact and parasitize a suitable host plant soon after germination [10], and access to a wider range of potential host plants should increase the likelihood of survival, regardless of the specific plants growing nearby [19]. Although less common than host plant specialists, many parasitic plant families do contain generalists, including some or all parasitic members of Orobanchaceae, Lauraceae, Convolvulaceae, Krameriaceae, and most of the 18 families of Santalales (sandalwoods, mistletoes and their relatives [20]).

If mutations that increase specialized feeding strategies increase in frequency when specific host resources are predictable [21], then traits associated with maintenance of generalist abilities are likely to decrease in frequency. If a generalist strategy involves the evolution of a generalpurpose suite of genes that are necessary and sufficient to successfully parasitize a wide range of hosts, then such a trend could lead to a long-term stable generalist strategy. Alternatively, if generalists maintain distinct sets of genes specific to different hosts, then the long-term maintenance of gene sets for attacking different hosts may be unlikely unless there is frequent reinforcement by a diverse range of hosts.

Triphysaria (Orobanchaceae) is a generalist parasite that feeds on a highly diverse collection of angiosperms in nature, including at least 30 species in 17 families of monocot and eudicot host plants [22]. We reasoned that sequencing transcriptomes from the haustorium of $T$. versicolor grown on distantly related hosts would maximize the potential to identify both shared and host-specific patterns of gene expression. The transcriptome datasets of $T$. versicolor provide a unique opportunity to leverage newly established genomic resources of the Parasitic Plant Genome Project (PPGP, [23]) with well developed functional protocols including parasite-host co-culture [9,24], haustorium induction assays [25], and parasite transformation [8,16,26]. By characterizing the molecular signature of host-parasite interactions, we stand to gain insight into the processes underway in a generalist parasite that facilitate a broad host range and learn about the molecular mechanisms that can facilitate the generalist parasite strategy.

Two substantial hurdles emerge when characterizing the transcriptomes of $T$. versicolor haustoria. The first is 
that gene expression profiles of specialized cells in the haustorium become diluted when harvesting even the tiny haustorium (1-2 $\mathrm{mm}$ diameter) of $T$. versicolor. The excellent histology and electron microscopy work by Heide-Jorgensen and Kuijt [12,13] revealed cells residing at the host parasite interface that had transfer cell-like morphology. The anatomy of these specialized cells includes dense cytoplasm, numerous small vacuoles, a highly invaginated cell membrane, and a labyrinthine cell wall (for a review see [27]). We hypothesized that the small collection of interface cells, including those with transfer-cell like morphology, facilitate the elusive molecular interaction between host and parasite, making them excellent candidates for transcriptome analysis. The second hurdle is that discovery of genes and subsequent gene expression analysis on a genome-wide scale is difficult without a sequenced and well-annotated genome, which is currently lacking for T. versicolor. Next Generation Sequencing (NGS) technologies have emerged as powerful tools for exploring new genomes because the cost per base is substantially lower than traditional dye-terminator or even pyro-sequencing (454) methods [28]. In the wake of the NGS revolution several tools for data analysis (for a review see [29]), including high performance de novo transcriptome assemblers like Trinity [30], have emerged to facilitate transcriptome analysis in uncharacterized model systems.

To overcome the limitations of reference independent transcriptome analysis of small numbers of difficult to harvest cells, we developed methods to sample parasitehost interface cells from $T$. versicolor grown on the distantly related and sequenced model hosts Zea mays (B73) (monocot) and Medicago truncatula (A17) (eudicot) via Laser Pressure Catapult Microdissection (LPCM). We extracted and then amplified exceedingly small RNA samples via T7-based linear amplification and then deeply sequenced each of the amplified parasite-host interface transcriptomes. We assembled millions of paired-end Illumina reads de novo, annotated each assembly and then estimated levels of gene expression via read mapping to the de novo assembled transcriptome. Using this approach, we identified genes that were part of a host-specific response as well as those that are part of a shared response of $T$. versicolor to the different hosts. We also verified the host-specific differential expression pattern of two Triphysaria expansin genes. Expansins are among the few genes known to be differentially regulated in haustoria $[31,32]$. Analysis of expansin genes allowed us to verify the differential gene expression pattern present in the interface sequence data, and demonstrate the first evidence that a $\beta$-expansin is highly upregulated in $T$. versicolor when grown on the $Z$. mays host. Our results suggest that the maintenance of a generalist feeding strategy in Triphysaria involves both generalized and specialized gene responses that help us understand Triphysaria's generalist feeding abilities.

\section{Results}

\section{Parasite host co-culture and microdissection of the} T. versicolor haustorium

T. versicolor and hosts were germinated and grown axenically in separate culture plates. To begin co-culture, hosts were transferred to fresh plates and $T$. versicolor were added and placed in close proximity $(\sim 1 \mathrm{~mm})$ to host roots. The attachment rate of $T$. versicolor to host roots was $\sim 90 \%$ for $M$. truncatula and $\sim 50 \%$ for $Z$. mays. This difference was likely due to the more rapid growth rate of $Z$. mays (compared to M. truncatula) coupled with the confined dimensions of the co-culture petri dish rather than differential parasite-host compatibility. Where host roots remained more or less stationary on the agar growth medium during early phases of co-culture, the attachment rates of $T$. versicolor were high (>90\%) and equivalent between $Z$. mays and $M$. truncatula.

The first step in sample preparation for LPCM was isolation and cryosectioning of haustoria formed on each host. The optimum section thickness was determined empirically by micro-dissecting samples from sections cut at $1 \mu \mathrm{m}$ section thickness intervals from $18-30 \mu \mathrm{m}$. For T. versicolor haustoria we determined that $25 \mu \mathrm{m}$ cryosections were optimal to allow efficient tissue release from the adhesive coated StarFrost ${ }^{\mathrm{TM}}$ LPCM slides coupled with maximum tissue harvest volume. Parasite and host cells that were in contact with each other at the interface were difficult to separate, so to ensure capture of the entire parasite interface cell population we intentionally included a minimal amount of host tissue, knowing that host transcripts could be identified and removed informatically. Figure 1 shows a typical haustorium cross section before (A) and after (B) LPCM.

To generate representative interface-cell samples we pooled 110 interface regions of interest (ROIs) from biological replicates ( $>8$ haustoria). The average ROI for T. versicolor interface transcriptome samples grown on M. truncatula was $54,910 \mu \mathrm{m}^{2}$ with a total area of 6.1 million $\mu \mathrm{m}^{2}$ that yielded $144 \mathrm{ng}$ total RNA. This pooled sample had an RNA integrity number (RIN) of 7.6, an $\mathrm{A}_{260} / \mathrm{A}_{280}$ of 1.58 and an $\mathrm{A}_{260} / \mathrm{A}_{230}$ of 0.76 . The average ROI for $T$. versicolor interface transcriptome samples grown on $Z$. mays was $56,079 \mu^{2}$ with a total area of 6.4 million $\mu \mathrm{m}^{2}$ that yielded $160 \mathrm{ng}$ total RNA with a RIN of 6.9, a $\mathrm{A}_{260} / \mathrm{A}_{280}$ of 1.68 and $\mathrm{a} \mathrm{A}_{260} / \mathrm{A}_{230}$ of 0.11 .

\section{Linear mRNA amplification from Laser Microdissected tissues}

The first step of T7 based amplification is cDNA synthesis with an oligo-dT/T7 RNA primer/promoter. It is critical that this step is highly efficient to minimize the bias toward 


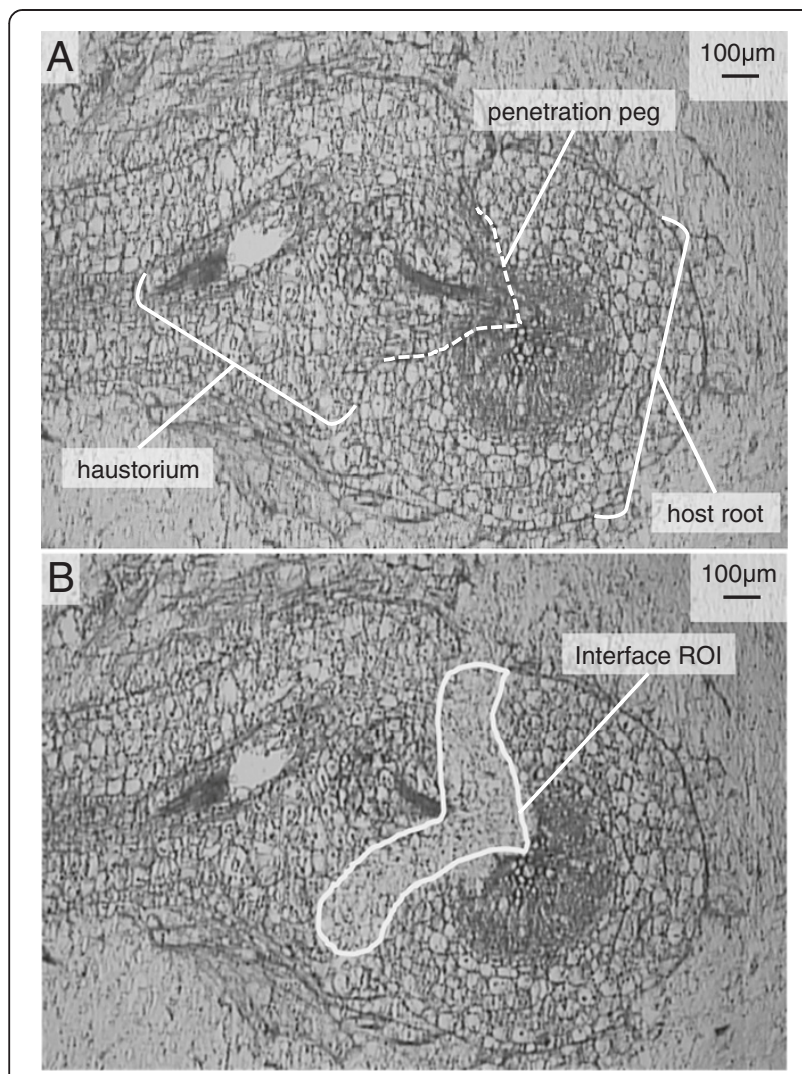

Figure 1 Laser Microdissected Haustorium. LPCM allows highly tissue- and cell-specific harvest after histological identification of tissues or cells of interest. A) Representative $25 \mu \mathrm{m}$ cross-section of T. versicolor haustorium on the host M. truncatula approximately 9 days post infestation, and prior to LPCM. The mature haustorium contains the xylem bridge that connects the parasite and host vasculature and is visible in the penetration peg.

B) The same section after LCPM shows the cleared interface tissue from the user-defined region of interest (ROI). The flakes of tissue are catapulted by a photonic cloud resulting from pulses of laser light focused between the tissue and glass slide. Multiple pulses of laser light raster across the $\mathrm{ROI}$ causing tissue in the selected region to be catapulted and then captured in the adhesive coated cap of a

$0.5 \mathrm{~mL}$ tube held by a robotic arm in very close proximity

$(<0.5 \mathrm{~mm})$ to the upper surface of the section affixed to the slide.

shorter fragment lengths in amplified samples [33]. We frequently observed a yellow-brown material at the host parasite interface that may have contributed to the low initial purity of the interface RNA samples, indicated by the low $A_{260} / A_{280}$ and $A_{260} / A_{230}$ ratios. Thus, we cleaned the interface total RNA with the Zymo ${ }^{\text {тм }}$ RNA Clean and Concentrator kit. Subsequently, we observed consistent amplification performance between technical and biological replicates of the cleaned interface total RNA as well as performance consistent with positive control samples of A. thaliana young leaf RNA of high quality and purity (28s/18s ratio: 1.9; RIN: 8; $\mathrm{A}_{260} / \mathrm{A}_{280}$ : 2.0, $\mathrm{A}_{260} / \mathrm{A}_{230}$ : 2.1).

Amplification of $\sim 100 \mathrm{ng}$ of total RNA routinely yielded 50-100 ug of amplified RNA (aRNA) after two rounds of amplification, which was consistent with the positive control (Arabidopsis young leaf total RNA), the expected performance of the Message Amp ${ }^{\text {TM }}$ II aRNA kit, and a previous report [34]. The aRNA yield after a single round of amplification was up to $100 \mathrm{ng}$, which is sufficient for construction of an Illumina sequencing library, yet we chose to amplify the samples for two rounds since it was desirable to have additional aRNA for further analyses including qRT-PCR validation of gene expression profiles. The fragment length profiles, as determined via Bioanalyzer $^{\mathrm{TM}}$, were reduced from the first to the second round of amplification, which is consistent with a previous report [33].

\section{Sequencing and assembly statistics}

Amplified interface RNA samples were sequenced on one lane each of Illumina's Genome Analyzer IIx with an $83 \times 83 \mathrm{bp}$ paired-end cycle protocol. Sequencing data are available at http://ppgp.huck.psu.edu [23]. The T. versicolor interface transcriptome datasets (Table 1 ) contained 17.9 million read pairs on $Z$. mays and 19.1 million read pairs on $M$. truncatula. Host reads from each interface transcriptome dataset were mapped to their respective host genomes, leading to the removal of 1.5 million $M$. truncatula reads and 0.4 million $Z$. mays reads from each respective transcriptome data set. Reads were quality trimmed and filtered (see methods), leaving $>26$ million reads (orphans and mate pairs) for each sample that were then assembled separately using Inchworm (Trinity, [30]) and post-processed to remove exact duplicate or non-translatable sequences. The interface transcriptome assembly of $T$. versicolor grown on $Z$. mays yielded $12.77 \mathrm{Mbp}$ of assembled sequence represented by 28,126 unigenes with an N50 of 525 bp (Table 1). The interface transcriptome assembly of T. versicolor grown on $M$. truncatula yielded $12.25 \mathrm{Mbp}$ of assembled sequence represented by 26,709 unigenes with an N50 of 536 bp (Table 1). Sequencing and assembly statistics were similar in all categories (Table 1) indicating that both data sets were of equivalent quality.

\section{Unigene annotation}

Unigenes were annotated using an objective classification of known plant genes from the PlantTribes 2.0 database [35,36] as described in Wickett et al. [37]. We assigned genes into a hierarchy of gene clusters, which includes approximate gene families (Tribes), and potentially narrower lineages (Orthogroups) which seek to represent descendants of a single ancestral gene in the collection of reference plant species [35-37]. We also classified unigenes from our experiment using BLAST to query sequence databases (Table 1 ). To identify host derived unigenes in the mixed-species transcriptomes, we established a pairwise nucleotide identity threshold 
Table 1 Read and assembly level statistics for $T$. versicolor interface transcriptomes

\begin{tabular}{|c|c|c|}
\hline \multirow[b]{2}{*}{ Sequencing } & \multicolumn{2}{|l|}{ Host plant } \\
\hline & Z. mays & M. truncatula \\
\hline Total raw sequence & 2.73 Gbp & $2.91 \mathrm{Gbp}$ \\
\hline Reads Total & $35,894,662$ & $38,228,134$ \\
\hline Host reads & $(401,352)$ & $(1,588,592)$ \\
\hline Host filtered & $35,493,310$ & $36,639,542$ \\
\hline Quality trimmed & $26,947,737$ & $27,325,845$ \\
\hline \multicolumn{3}{|l|}{ Assembly } \\
\hline Assembly length & $12.77 \mathrm{Mbp}$ & $12.25 \mathrm{Mbp}$ \\
\hline Unigenes Total & 28,126 & 26,709 \\
\hline Unigenes >500 bp & 9,369 & 9,718 \\
\hline Min/Max length (bp) & $197 / 3,115$ & $197 / 3,265$ \\
\hline $\mathrm{N}_{50}$ & $525 \mathrm{bp}$ & $536 \mathrm{bp}$ \\
\hline $\mathrm{N}_{50}>500 \mathrm{bp}$ & $731 \mathrm{bp}$ & $695 \mathrm{bp}$ \\
\hline \multicolumn{3}{|l|}{ Annotation } \\
\hline Host unigenes & $(4,967)$ & $(7,785)$ \\
\hline Non-plant unigenes & $(127)$ & (329) \\
\hline Triphysaria unigenes & 23,032 & 18,595 \\
\hline Triphysaria hits & 17,887 & 14,352 \\
\hline Other Plant hits & 2,975 & 2,086 \\
\hline No hits & 2,170 & 2,157 \\
\hline
\end{tabular}

Low quality reads were filtered before assembly, and host sequences were filtered both before and after assembly. Unigenes remaining after removal of host plant and non-plant sequences were aligned with BLASTx to sequences detected in any other PPGP transcriptome library of Triphysaria versicolor (http://ppgp.huck.psu.edu/). Unigenes with less than 95\% pairwise identity to either host or to other Triphysaria libraries were sorted further if a BLASTx search of the NR (www.ncbi.nlm.nih.gov) database yielded alignments of 1e-10 or stronger. The remaining unclassified unigenes were submitted to OrthoMCL DB and InterProScan. Unigenes that remained unclassified after the final screen are called "no hit" unigenes.

of $95 \%$ by querying a collection of $Z$. mays ESTs for $T$. versicolor grown on $M$. truncatula and vice versa (Additional file 1: Figure S1). The plot of T. versicolor unigene identity to each host database is clearly divergent at $95 \%$ compared to the reciprocal host database query. To verify that the incident high identity was not due to cross contamination between samples, we also queried the host EST databases with a de novo transcriptome assembly of Lindenbergia philippensis, a non-parasitic member of the Orobanchaceae [23]. The BLAST identity plot of the Lindenbergia transcriptome shows a similar trend to the plot of Triphysaria interface transcriptomes queried against the respective non-host databases (Additional file 1: Figure S1).

In order to remove host genes that may have escaped detection during pre-assembly read mapping, we further screened the assembly based on sequence similarity to cDNA (Phytozome, [38]) and EST databases (PlantGDB, [39]). This screen removed 4,967 unigenes from the transcriptome of $T$. versicolor grown on $Z$. mays and 7,785 unigenes from the transcriptome of $T$. versicolor grown on
M. truncatula (Table 1). The same reference transcripts used to screen the raw read data were also used to screen assemblies. The large number of putative host derived unigenes indicates that read screening with Mosaik at default values alone was insufficient to remove all host contamination. After the host screen the remaining unigenes were filtered for $T$. versicolor genes based on sequence similarity to genes detected in other PPGP libraries of Triphysaria versicolor [23]. We identified 17,887 unigenes from the transcriptome of $T$. versicolor grown on $Z$. mays and 14,352 unigenes from the transcriptome of $T$. versicolor grown on $M$. truncatula that had $>95 \%$ identity at the nucleotide level to $T$. versicolor genes from the other PPGP libraries. After removing unigenes with high similarity to $T$. versicolor unigenes from other assemblies in the PPGP database, the remaining 5272 unigenes in the interface transcriptome of $T$. versicolor grown on $Z$. mays and 4572 unigenes in the interface transcriptome of $T$. versicolor grown on $M$. truncatula were used to query the non-redundant protein sequence database (NR) at NCBI [40] using BLASTx at a threshold e-value of 1e-10. Roughly half of the remaining unigenes in each interface transcriptome had best hits to plants including the model species Arabidopsis, Populus, and Vitis, other Orobanchaceae, or $>30$ other plant species ("Other Plant Hits" Table 1).

Each T. versicolor interface transcriptome had $\sim 2300$ unigenes with no significant alignments to sequences in any of the above described external databases (Table 1). We took several additional steps to try to identify these unknown sequences. Though these unigenes are not classified by source, we identified potential plant gene orthologs for $\sim 20-25 \%$ of the remaining unigenes via the query of the PlantTribes 2.0 database. We then queried the extensive InterProScan (IPS) [41] and OrthoMCL DB [42] databases with the translated sequences of the remaining, unclassified unigenes. The majority of these unigenes $(>75 \%$ in each transcriptome) lacked significant similarity to genes in the OrthoMCL database, nor did they contain IPS peptide motifs (Additional file 2: Figure S2); they are thus referred to as "no hit" unigenes (Table 1). The scan of OrthoMCL DB resulted in identification of an additional 100 plant and 7 non-plant unigenes from the Zea grown T. versicolor and 85 plant and 16 non-plant unigenes from the Medicago grown T. versicolor (Table 1). Additionally, 370 Zea grown T. versicolor and 310 Medicago grown T. versicolor unigenes contained IPS motifs. Roughly half of the OrthoMCL DB hits lack descriptions or have minimal (e.g. one word) descriptions (Additional file 3). A similar pattern exists in the IPS search results, where about half of the unigenes with IPS motifs contain only putative secretion signals and/or transmembrane domains (Additional file 3). Overall our efforts to classify the unigenes in each assembly resulted in identification of potentially 
orthologous sequences for $82 \%$ of the Zea grown $T$. versicolor interface unigenes and $88 \%$ of the Medicago grown $T$. versicolor unigenes. We were able to assign a putative origin to $>90 \%$ of unigenes in both transcriptomes and only $5 \%$ in each transcriptome remain unclassified. Of these, 493 unigenes from the $T$. versicolor grown on Medicago assembly and 536 unigenes from the T. versicolor grown on Zea assembly are longer than $300 \mathrm{bp}$ and have read support.

The interface transcriptome of $T$. versicolor grown on $Z$. mays and $M$. truncatula contained a total of 127 and 329 unigenes, respectively, with best hits to non-plant species (Table 1). The non-plant component of each interface transcriptome included best hits to 16 taxa shared by both interface libraries. These included Escherichia, Aspergillus, Clavispora, Burkholderia, and others. Among this set, Burkholderia was the most highly represented taxon ( $>20$ fold increase over any other species) in the non-plant component of both interface transcriptomes. These interface Burkholderia sequences were not detected in the reference transcriptome (TrVeBC1, sequence identity cutoff $>90 \%$ ).

The remaining "no hit" sequences, especially those $>300$ bp with read support, could represent unannotated host or parasite genes, uncharacterized associated symbionts, or incidental contamination. All remaining unigenes not assigned to a host plant or non-plant source in the NR database $(23,032$ for $T$. versicolor on $Z$. mays and 18,595 for $T$. versicolor on $M$. truncatula) are considered collectively as "putative T. versicolor derived unigenes."

\section{Comparative interface transcriptome profiles of $T$. versicolor}

To examine the profiles of the $T$. versicolor interface transcriptomes, we used an annotated transcriptome from above ground tissues of autotrophically grown $T$. versicolor as a reference ([23] Triphysaria assembly TrVeBC1). We sorted unigenes by (PlantTribes 2.0) Orthogroups to identify host-specific and shared components of the interface transcriptomes of $T$. versicolor grown on $Z$. mays and $M$. truncatula (Figure 2). As expected, the largest number of Orthogroups $(5947$, or $53.6 \%$ of the total detected in $T$. versicolor) were shared between all three transcriptomes, and likely represent expression of genes involved in processes common to a wide variety of cell types. A large number of Orthogroups (1124) were shared between the interface transcriptomes of $T$. versicolor interacting with both hosts. These genes likely include a putative core set of parasite genes that are active irrespective of the host plant species. Many additional Orthogroups were either exclusive to the interface and host-specific (677 for Z. mays and 361 for $M$. truncatula), or shared with above ground phases of growth (1066 for Z. mays and 314 for M. truncatula).

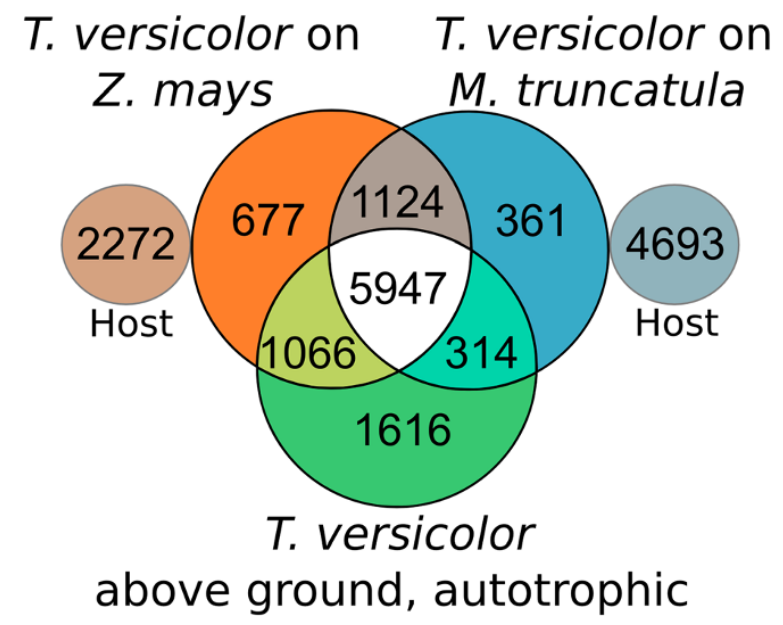

Figure 2 Transcriptome Orthogroup Venn. Venn diagram showing the number of Orthogroups in the interface transcriptomes of $T$. versicolor with hosts $Z$. mays and $M$. truncatula and an above ground, autotrophically grown T. versicolor transcriptome (TrVeBC1) constructed from leaves, stems and inflorescences. Also shown are the numbers of host-derived Orthogroups. The lack of overlap between host and parasite transcriptomes does not imply lack of shared Orthogroups, but indicates the total number of host Orthogroups for a point of comparison.

Our annotation strategy includes assignment of a GO Slim category term derived from the best BLAST hit in PlantTribes 2.0. GO Slim categories are the broadest designations of GO and are useful for transcriptome-wide comparisons. The host-specific component of the T. versicolor interface transcriptome is likely to contain genes that interact with unique aspects of host biology while those that are shared likely contain genes essential for parasitism. To determine if the annotation profiles were similar between the overlapping and unique transcriptome components we plotted the proportion of GO Slim categories of unigenes (Figure 3) represented by unique or overlapping Orthogroups in Figure 2. GO Slim category profiles between equivalent components of each interface transcriptome were generally similar to each other, yet often distinct from profiles of non-equivalent components of each interface transcriptome (Figure 3). For instance, interface-unique Orthogroup profiles were similar between both interface transcriptomes, yet distinct from the above ground Orthogroup profiles of both interface transcriptomes.

GO Slim category profiles in overlapping and unique sets of Orthogroups within each interface transcriptome were tested for proportionality by a Chi-Square test (Additional file 4: Figure S3A-F). The results of all 6 tests showed disproportionality and were strongly significant $(\mathrm{P}<<0.0001)$. The number of unigenes in each GO Slim category, with strong residual values (strongly positive or strongly negative, thus disproportionate), is indicated in Additional file 4: 

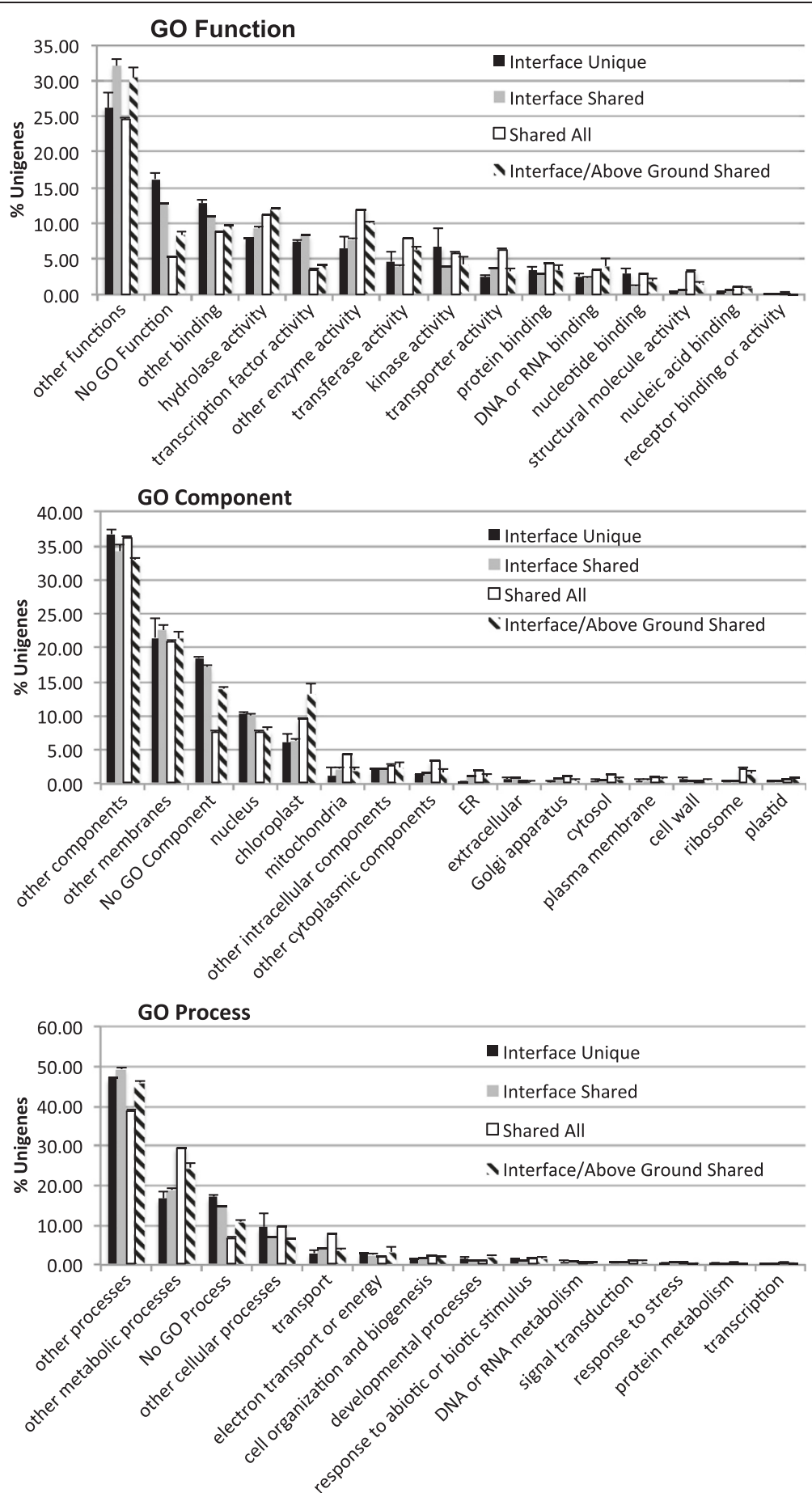

Figure 3 GO Slim category summary. GO Slim category terms of unigenes in interface transcriptomes of T. versicolor and the above ground reference assembly of $T$. versicolor. Each series displays the average number of unigenes in equivalent transcriptome components with a given GO Slim term. For instance, "Interface Unique" indicates the average number of unigenes from interface unique components in both Medicago and Zea grown T. versicolor transcriptomes. Error bars are standard error of the mean. "Interface Unique" = unigenes from Orthogroups that are host and interface specific, "Interface Shared" = unigenes from Orthogroups that are interface specific and shared between interface transcriptomes, "Shared All" = unigenes from Orthogroups shared between both interface transcriptomes and the above ground transcriptome, "Interface/Above Ground Shared" = unigenes from Orthogroups that are shared between the above ground, autotrophic transcriptome and the host-specific interface transcriptome. 
Figures S3A-F. The results of this analysis are concordant with the plot of GO Slim category profiles (Figure 3). The most striking result is the consistently strong overrepresentation of unigenes lacking GO Slim categories in interface-unique Orthogroups (Additional file 4: Figure S3A-C: columns A and B, Figure S3D-F: columns E and F) compared to the consistent weak representation of unigenes lacking GO Slim categories in the shared Orthogroups (Additional file 4: S3A-C: column D, Figure S3D-F: column H). Interestingly the Orthogroups shared in the interaction of $T$. versicolor with both hosts are overrepresented by "transcription factor activity" GO Slim Function terms (Additional file 4: Figure S3A: column B and 3D: column F) and underrepresented by "transport" GO Slim Process terms (Additional file 4: Figure 3: column B and 3F: column F). This indicates that there are transcription factor genes active at the parasite-host interface that are not expressed in the above ground reference transcriptome. In contrast, the transporter gene families expressed at the interface are expressed in the above ground reference transcriptome as well.

\section{Highly expressed genes at the host-parasite interface}

An advantage of using a de novo assembled transcriptome for RNA-Seq is an intrinsic threshold for transcript detection. If the transcript is represented by sufficient reads for de novo assembly, the presence of a target for de novo RNA-Seq is evidence for the presence of a transcript. The reference assembly TrVeBC2 [23] includes data from the haustorium of $T$. versicolor grown on $M$. truncatula and was used as a reference to map reads from each interface transcriptome. We correlated normalized reads (reads/ kilobase/million mappable reads (RPKM)) from unigenes belonging to Orthogroups shared between the interface transcriptomes and the above ground reference transcriptome, TrVeBC1 (Additional file 5: Figure S4). For unigenes detected in both interface transcriptomes the correlation was high (Pearson's $\mathrm{R}=0.81$ ), which indicates low technical and biological variability between the interface transcriptomes.

To determine the expression level of each unigene we also mapped reads to each respective interface de novo assembly. The 20 most highly expressed unigenes in each set of shared and unique Orthogroups from the two transcriptomes are presented in Additional file 6: Figures S5A-C. We queried this set of 120 unigenes against the NR database using BLASTx (e-value threshold: 1e-10) and 17 annotated plant genomes using BLASTn and BLASTx. The results of the database queries using BLAST are presented in Additional file 6: Figures S5A-C. The besthit descriptions from searches in NR were concordant with the annotations assigned using PlantTribes 2.0 (See BLAST results in Additional files 7 and 8). We also used InterProScan to predict signal peptides and transmembrane domains for the unigenes listed in Additional file 6: Figure 5A-C (Additional files 9 and 10). The motif prediction tools frequently identified putative transmembrane domains in unigenes annotated as transporters and secretion signals in unigenes annotated as secretory proteins. Of the 120 unigenes listed (Additional file 6: Figures S5A-C), nine had no hit when queried against NR. The remaining unigenes had best hits to plant species. About 30\% of these 120 unigenes have either no BLAST hits in NR or align to predicted, hypothetical, or otherwise uncharacterized sequences. This result is consistent our finding that the interface is enriched with unigenes that lack GO Slim category assignments (thus functional annotations).

Among the most highly expressed genes in the shared orthogroups of interface samples of $T$. versicolor grown on in both $Z$. mays and $M$. truncatula (Additional file 6: Figure $\mathrm{S} 5 \mathrm{~A}$ ) are a $\beta$-expansin gene (see below), genes for several other cell wall modifying enzymes, and a gene encoding a putative ap2-erf domain transcription factor. A striking pattern in the shared interface Orthogroups was 10 unigenes (including six of the most strongly expressed unigenes from T. versicolor grown on Medicago) with sequence identity to annotated pathogenesis-related proteins in other eudicot species. A single $M$. truncatula unigene passed through the host plant removal process in the common interface component (ID 5537); this also shared high sequence identity with a pathogenesis-related protein.

Of the unigenes listed in Additional file 6: Figures S5A-C, 42 from the interface transcriptome of $T$. versicolor grown on $Z$. mays and 34 from the interface transcriptome of $T$. versicolor grown on $M$. truncatula had strongest BLAST hits to Asterid genomes (including Mimulus guttatus). When we queried the 17 plant genomes database there were slightly more best hits to legumes in the Medicago grown Triphysaria data set, perhaps because there is less sequence divergence between the eudicots Triphysaria and Medicago than between the more distantly related Triphysaria and Zea. This results in a somewhat broader range of ambiguous sequence identity between host and parasite. Despite rigorous filtering, a single putative $Z$. mays transcript and three putative $M$. truncatula transcripts persevered (indicated in bold) in the highly expressed gene list in Additional file 6: Figure S5.

\section{A novel $\beta$-expansin is differentially expressed at the parasite-host interface}

Among the highly expressed unigenes observed in the interface transcriptome of $T$. versicolor grown on $Z$. mays was a putative $\beta$-expansin (Additional file 6: Figure S5A). Manual curation of the read mapping data indicated that it was highly expressed when grown on $Z$. mays, and a nearly identical unigene from the $M$. truncatula-grown Triphysaria interface was lowly expressed. This apparently 
host-specific gene expression pattern was of interest because expansins are cell-wall loosening proteins (for a review, see [43]) that have been implicated in the interaction between parasitic plants and their hosts [31,32]. While the $\beta$-expansin gene was expressed in both samples, read mapping evidence suggested that this gene (unigene 772) was highly differentially expressed. As a point of comparison, we investigated a putative $\alpha$-expansin, unigene 11, which showed a reciprocal pattern of high expression in the interface transcriptome of $T$. versicolor grown on $M$. truncatula. We verified the nucleotide sequence of unigenes 772 and 11 via dye-terminator sequencing of PCR products amplified from interface aRNA.

Phylogenetic analysis of $\beta$-expansin unigene 772 shows that it is nested within a supported clade of dicot $\beta$-expansin sequences (Additional file 11: Figure S6A) indicating that unigene 772 is a dicot $\beta$-expansin and not a $Z$. mays derived sequence. Annotation via InterProScan supports an expansin identity for 772 (Additional file 9) and shows a putative 5 ' signal peptide (Additional file 6: Figure S5A), consistent with a role in the apoplast that is typical for expansins. Additionally, the results of all of the BLAST searches suggest that unigene 772 is a $T$. versicolor derived sequence. Phylogenetic evidence for unigene 11 does not yield a well-resolved tree of $\alpha$-expansins (Additional file 11: Figure S6B), but the BLAST results suggest that the pairwise nucleotide identity to known, or putative (e.g. ESTs) M. truncatula genes is $<70 \%$, while unigene 11 has high identity ( $>95 \%$ pairwise nucleotide identity) to Triphysaria unigenes in other PPGP assemblies.

\section{Quantitative Real-Time PCR verification of host specific expansin expression}

We sought to verify the reciprocal expression patterns of these two expansins via qRT-PCR. Unigenes 772 and 11 were assigned formal names TvEXPB1 and TvEXPA4, respectively. We verified that primers were specific to their targets by melting curve analysis. To further verify that the TvEXPB1, TvEXPA4, and TvActin primers were specific to parasite transcripts, we harvested portions of host roots that were immediately adjacent to mature $T$. versicolor attachments and interrogated them via qRT-PCR. In these host root samples we were able to detect ZmActin in Z. mays root samples and MtActin in M. truncatula samples, while the parasite primers yielded signal consistent with background.

We interrogated biological replicates of the T. versicolor host-parasite interface cells grown on both $Z$. mays and M. truncatula via qRT-PCR for expression of the reference gene, TvActin, TvEXPA4, and TvEXPB1 (Figure 4). TvEXPB1 is up-regulated $>120$ fold $(\mathrm{P}=0.024)$ in $T$. versicolor haustorial interface cells grown on $Z$. mays relative to $T$. versicolor grown on $M$. truncatula.

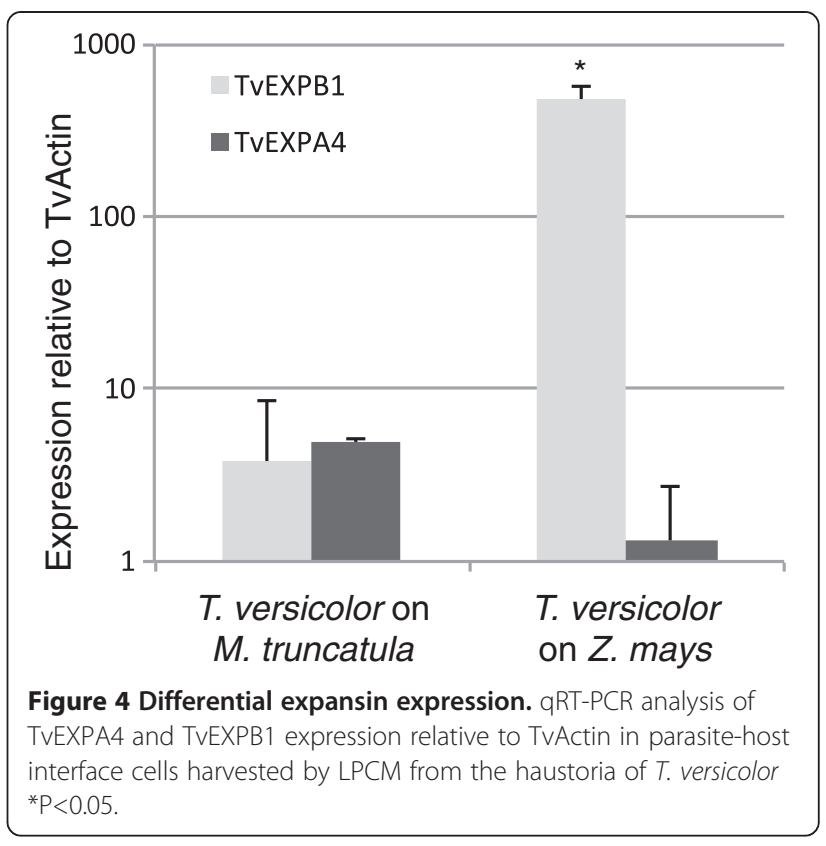

TvEXPA4 shows a weak reciprocal pattern $(\mathrm{P}=0.17)$. The expression patterns of TvEXPA4 and TvEXPB1 are concordant with our de novo RNA-Seq results. Additionally, when we examined TvEXPB1 expression in whole haustorium samples the signal was indistinguishable from background, suggesting that the massive upregulation of TvEXBP1 is specific to a small number of interface cells.

\section{Discussion}

Using a workflow that allowed us to sample, sequence, and de novo assemble transcriptomes from cells at the hostparasite interface we have shown that $T$. versicolor expresses genes in a host specific manner. This preliminary look at genes expressed at the parasitic plant-host plant interface suggests that the basis for generalist parasitism is constituted, at least in part, by host-specific patterns of gene expression. Generally, this work demonstrates the potential to discover genes de novo and examine genome-wide patterns of gene expression in a highly tissue-specific manner in organisms that lack a sequenced genome.

\section{Laser Microdissection (LM) is a powerful tool for plant transcriptomics}

The power to develop a comprehensive picture of any biological system lies with understanding the myriad processes underway in complex organs and tissues. A primary hurdle to revealing this picture is the ability to identify, separate, capture and analyze tissues and cells of interest. Several authors have emphasized the importance of high resolution, high through-put investigations of gene expression in a tissue- and cell-specific manner as well as the need to survey gene expression in a global manner, and why LM 
(including LPCM) is emerging as a powerful tool for genomics [44-47].

LM generated samples from some model systems have been examined using microarrays [48-53] allowing investigators to analyze global gene expression patterns in specific tissues and cell types. More recently, LM has been increasingly coupled with NGS to sequence the transcriptomes of various tissues in Z. mays [54-56] and S. lycopersicum [34]. The advent of de novo transcriptome assembly now makes global surveys of gene expression in specific tissues and cells of non-sequenced organisms a logical next step. To examine the parasite-host interface transcriptome of $T$. versicolor, we combined LPCM with robust T7 based linear RNA amplification [33,57-60] in concert with Illumina mRNA-Seq, high performance de novo transcriptome assembly [30], and various assembly post-processing tools.

For the haustorium of $T$. versicolor our sampling strategy was based largely on detailed histology and transmission electron microscopy work done with field-collected specimens [12,13]. This critical background information allowed us to identify cells of interest within the haustorium and then subsequently identify regions of the haustorium in cryosections that contained these cells. Plant tissues must be embedded prior to LPCM and preparatory steps can have an impact on the quantity and quality of RNA preparation [61]. However, the ability to identify tissues of interest must be balanced with downstream usability. The histological quality of the section is an important consideration that may determine the sample preparation method. Paraffin embedded sections generally provide high histological quality at the expense of RNA quality and yield $[62,63]$. Histological quality increases with thinner sections for sampling at a finer spatial resolution and the efficiency of pressure catapulting increases with thinner sections, yet Kerk et al. [64] report increased RNA yield from thicker sections. We found that the optimum section thickness for capturing interface cells of the T. versicolor haustorium was 20-25 $\mu \mathrm{m}$. This was determined based on a balance of our ability for histological identification of tissues and cells of interest with efficient tissue release from the slide during the pressure catapult phase of LPCM. The integrity of plant tissues that are susceptible to damage by flash freezing can be preserved by infusion with a cryoprotectant [65]. Cryosectioning with the CryoJane ${ }^{\mathrm{TM}}$ (a cryosection transfer system) allowed us to easily capture serial cryosections of T. versicolor haustorium that routinely yielded high quality RNA from carefully chosen samples.

By design, our sampling strategy minimized the likelihood that differences in gene expression arose from temporal or spatial sampling artifacts. The co-culture of $T$. versicolor is not highly synchronous, so our sample of haustoria represents a broad temporal window of connections that are $\sim 8-10$ days old. We collected $\sim 110$ interface ROIs which diminishes the likelihood of spatial sampling artifacts. Furthermore, highly similar statistics throughout sample processing and data analysis, including a high correlation of read counts for unigenes in shared Orthogroups (Additional file 5: Figure S4) and verification of the expression pattern of TvEXPB1 in additional experiments with biological replicates (Figure 4), indicates low variation from either biological or technical sources.

\section{Parasite-host interface transcriptomes help to identify core parasite genes}

Orobanchaceae include the pernicious weeds Striga, Orobanche and Phelipanche, as well as the model parasite Triphysaria. We reasoned that by discovering a subset of genes that likely include those central to the response of $T$. versicolor to distantly related hosts, we would move closer to identifying a core set of parasitism genes operating in the weedy Orobanchaceae. The molecular dialogue of the parasitic plant with its host is largely uncharacterized [66]. Here we report the identification of the subset of genes expressed at the host-parasite interface by $T$. versicolor in response to both of the distantly related hosts $Z$. mays and $M$. truncatula. Additionally, we identified host-specific patterns of gene expression indicating that the generalist parasitic plant $T$. versicolor maintains suites of genes for use with different hosts.

The genes of $T$. versicolor expressed at the host-parasite interface in response to both $Z$. mays and $M$. truncatula included a substantial set of genes annotated in other plant species as pathogenesis- (or pathogen-response) related proteins. This included six of the most highly expressed genes in the Triphysaria interface grown on M. truncatula, and three when grown on $Z$. mays, but a number of additional unigenes were also putative homologs of genes that are upregulated during pathogen invasion (dirigent-like, acidic endochitinase, disease resistance proteins, etc.). While upregulation of genes of these classes would be expected as a defense response to pathogens, this observation suggests that pathways commonly involved in plant protection are also turned on by the parasite during the process of host invasion. It has been already suggested that parasitic plants may have recruited pathways from allelochemical detoxification for use during haustorium signal transduction [67]. Whether Triphysaria is defending itself during the invasion process with pathogen resistance (PR) genes, or has recruited PR pathways as offensive 'weapons' is not yet clear, but it does appear that a portion of the core gene set for Triphysaria parasitizing either host has been derived from pathways that plants use for defense against pathogens.

There is no consensus for why true generalist parasites occur or persist, even infrequently, despite evidence for an evolutionary trend toward specialization ([18] and references there-in). We hypothesized that a successful 
generalist feeding strategy might rely heavily on generalpurpose genes that were deployed for feeding on any host. Instead, we see large components of the interface transcriptome that are detected only when feeding on one of the tested host plants. Of the 2162 Orthogroups detected only in interface transcriptomes, about half (52\%) were expressed on both host species, but a substantial fraction of interface Orthogroups were detected only when grown on maize (31\%), or on Medicago (17\%). Long-term maintenance of extensive genetic machinery directed toward parasitism of subsets of possible hosts will require maintenance of selective pressures on the genes that are deployed in a host-specific manner. Unless the parasite routinely encounters and parasitizes a wide range of host plants, selective pressure on some host-specific genes will be relaxed and the gene functions eventually lost, limiting the plant's potential to parasitize some hosts, despite the potential advantages of a large host range. The evidence for host specific gene expression suggests that $T$. versicolor regularly parasitizes across a broad host range, maintaining selective pressure on genes used in a host-specific manner. Indeed the advantage of a broad host range has been explored where host populations are moderately variable in space and time [19]. This study serves as a starting point for a broader survey of putative and potential hosts for Triphysaria as well as a first look at gene expression profiles of highly specialized tissues in parasitic plant haustoria.

It is intriguing that the host-specific and shared-interface gene families (Orthogroups) were over-represented by unigenes with no GO Slim category term assignment compared to the above ground transcriptome. Because Orthogroups were defined based on a classification of sequenced and annotated plant genomes [35-37] to which the parasite genes were assigned, this observation highlights the fact that genes expressed in the haustorium include many that have been recruited from the subset of genes whose function is not yet known in any plant. In addition, approximately 1300 unigenes from each interface transcriptome lack strong homology to any known sequence, though $25 \%$ of these unigenes are high identity reciprocal best hits in the interface data sets from each host. Further, because such patterns are reproducible in the interface transcriptomes of $T$. versicolor when grown on different host plants, these data suggest that genes of unknown function are expressed in the haustorium in a host-specific manner. Our data also suggest that underground phases of growth in $T$. versicolor are enriched for genes of unknown function.

Of those unigenes with GO Slim category assignments, the shared interface-specific Orthogroups are overrepresented for the GO Slim Function category "transcription factor" and underrepresented for the Go Slim Process category "transport." This indicates that there are transcription factor Orthogroups unique to the interface (relative to the above ground reference transcriptome), yet Orthogroups involved in transport processes are active in all three transcriptomes examined. The latter observation regarding transport does not rule out differential expression of particular genes that are expressed in all three transcriptomes in this study.

\section{TvEXPB1 encodes a $T$. versicolor $\beta$-expansin that is part of} a host-specific response

The expansin gene family includes 4 main groups: $\alpha, \beta$, expansin-like A and expansin-like B [43]. Expansins are thought to loosen plant cell walls by allowing slippage of cell wall polymers [43]. Their activity is non-enzymatic, but they are distantly related to glycoside hydrolase family proteins [43]. Expansins are involved with cell growth [68] and have been implicated in the interaction of bacterial plant pathogens $[69,70]$, plant-parasitic nematodes [71,72] and parasitic plants $[31,32]$ with their plant hosts. In each each case, expansins are suggested to play a role in host invasion.

Expansin activity has been assayed in the cell walls of both monocots [73-75] and dicots [76,77]. $\beta$-expansins have activity that is specific to the cell walls of grasses, but not dicot or most other monocot cell walls [78]. The reverse pattern of action is found for $\alpha$-expansins, which suggests substrate specificity for both $\alpha$ - and $\beta$-expansin proteins $[43,79]$. Typically, expansins are found at low concentrations [43] with the exception of grass pollen that secretes massive amounts of $\beta$-expansins [76] that likely serve to loosen stylar tissue during pollen tube growth $[80,81]$. Although the exact mechanism of action remains unknown, grass cell walls have relatively small amounts of xyloglucan and pectin; these are replaced with $\beta-(1 \rightarrow 3)$, $(1 \rightarrow 4)$-D-glucan and glucuronoarabinoxylan. Both of these grass cell wall components are potential targets of $\beta$-expansins in their wall-loosening activity [82]. Throughout more than a decade of research, the accumulation of $\beta$-expansins to high levels was thought to be specific only to grass pollen [82].

In this study, we have shown that the transcript level for the $\beta$-expansin TvEXPB1 is among the most highly expressed genes in the interface transcriptome of $T$. versicolor grown on $Z$. mays. Relative to the interface transcriptome of $T$. versicolor grown on $M$. truncatula, the expression of TVEXPB1 is greatly up-regulated ( $>120$-fold) in the parasite-host interface tissues of $T$. versicolor grown on $Z$. mays. The massive and host-specific expression of TvEXPB1 is suggestive of a role in a dicot parasite's interaction with a grass host. Additionally, the signal for TVEXPB1 in whole haustorium samples of $T$. versicolor grown on $Z$. mays was undetectable relative to the interface samples suggesting that TVEXPB1 is highly specific to the host-parasite interface. 
Taken together, the evidence for grass cell wall-specific activity of $\beta$-expansins and the massive upregulation of a parasite $\beta$-expansin at the parasite-host interface suggests that $T$. versicolor expresses TvEXPB1 when interacting with the monocot host $Z$. mays in an effort to manipulate host cell walls. Heide-Jørgensen and Kuijt [12,13] observed that host cortical and epidermal cells seemed crushed and displaced at the host parasite interface in the haustorium of $T$. versicolor. The mechanism of host tissue displacement may include cell wall modifying proteins like expansins that have host cell wall-specific activity. Such a mechanism would allow the parasite to soften or separate host cell walls without affecting the integrity of its own cell walls in the penetrating haustorium. The specific role that expansins play in the host parasite interaction will only be uncovered through detailed functional analysis. This includes focused gene expression analysis, targeted silencing of $T$. versicolor expansin genes and biochemical characterization to determine the substrate specificity of the expansin proteins encoded by these genes.

\section{Are a ubiquitous genus of soil bacteria symbionts of $T$. versicolor?}

The best represented genus in the non-plant transcriptome component ( $\sim 60 \%$ of non-plant hits in T. versicolor grown on $Z$. mays and $\sim 45 \%$ non-plant hits in $T$. versicolor grown on $M$. truncatula) was Burkholderia, a common genus of soil bacteria. The high frequency of hits to this bacterium is surprising for three reasons: (1) the relative frequency of other non-plant genera was much lower, (2) T. versicolor seeds were aggressively surface sterilized prior to axenic co-culture, and (3) Burkholderia-derived unigenes were not detected in the above ground reference assembly. The low frequency of hits to other genera (including plant pathogenic fungi, human and other bacteria) could be explained by incidental contamination from the lab environment, however the preponderance of Burkholderia hits only in the interface samples suggest the presence of an organism belonging to this genus in the co-culture system. The unigenes were generally $<1 \mathrm{kbp}$ (indicating transcript-sized unigenes) and RNA samples were DNase treated, diminishing the likelihood that these unigenes originated from genomic DNA contamination.

The genus Burkholderia has received increasing attention in the last two decades due in part to a diverse catalog of host interactions ranging from human pathogen to plantgrowth promoting rhizosphere fauna [83]. Of particular interest here is their potential role as beneficial plant endosymbionts [83]. Attsat [84] noted the presence of filamentous bacteria-like structures in haustorial cells and that application of terramycin significantly reduced haustorium formation in Orthocarpus purpurascens (syn. Castilleja exserta), a hemi-parasite that is a close relative of T. versicolor [85]. The presence of Burkholderia in an axenic co-culture system points to an intriguing possibility: a species of Burkholderia was carried through surface sterilization with the seeds of $T$. versicolor. Evidence that may suggest a role for a prokaryotic endosymbiont in parasitic plant biology [84] considered with evidence for the presence of a genus of beneficial soil bacteria hints at a symbiosis between Burkholderia and Triphysaria. The presence and possible roles of Burkholderia at the hostparasite interface in $T$. versicolor are currently under investigation.

\section{Conclusions}

Triphysaria represents an important asset in the identification of genes and evolutionary processes that are central to parasitic plant biology, and one that is key to the development of new control strategies for the weedy Orobanchaceae. Highly tissue-specific transcriptome analysis in Triphysaria versicolor, an experimental model parasitic plant that has yet to have its genome sequenced, has revealed host-specific gene expression. Furthermore, genes represented at the parasite-host interface are enriched for genes of unknown function relative to above ground phases of growth. Ongoing development of molecular techniques, including parasitic-plant transformation $[8,86]$ will facilitate the functional characterization of genes whose roles may be central in the parasitic plant-host plant interaction.

\section{Methods}

\section{Growth of plant material Triphysaria versicolor}

Seed was collected from hundreds of open pollinated plants growing in coastal grassland stands of Napa California laboratories (University of California, Davis, USA) from the same source population as our prior transcriptome sequencing studies [37,87]. Seeds were surface sterilized in $70 \%$ ethanol for $10 \mathrm{~min}$. while gently shaking, and then washed $3 x$ with sterile distilled $\mathrm{H}_{2} \mathrm{O}$. Seeds were further sterilized and scarified by a wash with a $50 \%$ bleach $+0.01 \%$ Triton $\mathrm{X}-100$ (Sigma) solution for $30 \mathrm{~min}$. while gently shaking. Seeds were then washed $10 x$ with sterile distilled $\mathrm{H}_{2} \mathrm{O}$ and placed in petri dishes containing co-culture medium (1/4x Hoagland's basal salt and nutrient mix, $7.5 \mathrm{~g} / \mathrm{L}$ sucrose, $6 \mathrm{~g} / \mathrm{L}$ plant tissue culture grade agarose, $\mathrm{pH}$ of 6.1 ) and wrapped with Parafilm ${ }^{\mathrm{TM}}$. Seeds were stratified for 4 days at $4^{\circ} \mathrm{C}$ in the dark then transferred to a $16^{\circ} \mathrm{C}$ growth chamber under a 12-hour light regime with a light intensity of $30 \mu$ moles photons $/ \mathrm{m}^{2} / \mathrm{sec}$. T. versicolor seedlings were grown for 14-17 days then transferred to fresh co-culture plates with hosts.

\section{Medicago truncatula (A17)}

Seed was generously provided by Zengyu Wang (Noble Foundation, Oklahoma, USA). Seeds were scarified by 
incubation with occasional stirring in $18 \mathrm{M} \mathrm{H}_{2} \mathrm{SO}_{4}$ for 8 min., and then washed $5 \times$ with sterile distilled $\mathrm{H}_{2} \mathrm{O}$. Seeds were surface sterilized by a wash with $50 \%$ bleach $+0.01 \%$ Triton X-100 (Sigma) for $3 \mathrm{~min}$. while gently shaking. Seeds were washed $10 \mathrm{x}$ with sterile distilled $\mathrm{H}_{2} \mathrm{O}$ and placed in a $50 \mathrm{~mL}$ conical bottom tube with $25 \mathrm{~mL}$ of sterile distilled $\mathrm{H}_{2} \mathrm{O}$ at $25^{\circ} \mathrm{C}$, in the dark, over-night while gently shaking. The next day seedlings were transferred to sterile filter paper (Whatman \#5), moistened with sterile distilled $\mathrm{H}_{2} \mathrm{O}$, and placed in petri dishes. Seedlings were placed at $25^{\circ} \mathrm{C}$, in the dark, over-night. Seedlings were transferred to coculture medium and wrapped with Parafilm ${ }^{\mathrm{TM}}$ then placed in a growth chamber at $25^{\circ} \mathrm{C}$ under a 16-hour light regime with a light intensity of $100 \mu$ moles photons $/ \mathrm{m}^{2} / \mathrm{sec}$ for 5-7 days, then transferred to fresh co-culture plates with parasites.

\section{Zea mays (B73)}

Seed was generously provided by David Braun (University of Missouri, Columbia, USA). Seeds were surface sterilized by a wash with $20 \%$ bleach $+0.08 \%$ Triton X-100 (Sigma) for 10 min. while gently shaking. Seeds were washed $3 x$ with sterile distilled $\mathrm{H}_{2} \mathrm{O}$. Seeds were further surface sterilized by a wash with $70 \%$ ethanol for $5 \mathrm{~min}$. while gently shaking. The ethanol was decanted and the seeds were left to air dry in a laminar flow hood. Dry seeds were placed in petri dishes containing co-culture medium and wrapped with Parafilm ${ }^{\mathrm{TM}}$ then placed in a growth chamber at $25^{\circ} \mathrm{C}$ under a 16-hour light regime with a light intensity of $100 \mu$ moles photons $/ \mathrm{m}^{2} / \mathrm{sec}$ for 5-7 days, then transferred to fresh coculture plates with parasites.

\section{Parasite and host co-culture}

Zea mays or Medicago truncatula were transferred to fresh co-culture medium at the times indicated above. Host roots were carefully oriented to allow placement of $T$. versicolor (grown as described above) seedlings in close proximity to host roots, with the parasite root tips 0.5 $1 \mathrm{~mm}$ from the host root.Co-culture plates were sealed with Micropore $^{\mathrm{TM}}(3 \mathrm{M})$ surgical tape and placed in a growth chamber at $25^{\circ} \mathrm{C}$ under a 16 -hour light regime with a light intensity of $100 \mu$ moles photons $/ \mathrm{m}^{2} / \mathrm{sec}$ for 8-10 days.

\section{Tissue processing and sample preparation for sequencing Tissue harvest}

After 8-10 days co-culture, the haustoria of T. versicolor were harvested by making cuts in both the host root and parasite root $\sim 1-2 \mathrm{~mm}$ adjacent to the haustorium under a stereomicroscope and were embedded in Shandon Cryomatrix $^{\mathrm{TM}}$ (Thermo Scientific) dispensed in a $10 \mathrm{~mm} \mathrm{x}$ $10 \mathrm{~mm}$ x 5 mm Cryomold ${ }^{\mathrm{TM}}$ (Tissue-Tek). Dissected tissue was quickly oriented with the host root on the vertical axis and with all haustoria equidistant from the bottom of the mold. Samples were quickly frozen on dry ice and stored at $-80^{\circ} \mathrm{C}$.

\section{Cryosectioning and dehydration}

Embedded haustoria were sectioned in a Cryotome SME (ThermoFisher Scientific) at 20-25 $\mu \mathrm{m}$. Frozen sections were mounted to slides using the Cryo-Jane ${ }^{\text {тм }}$ Tape Transfer System (Leica Microsystems). Mounted sections were dehydrated in a series of organic solvent baths (RT 70\% ethanol (10 $\mathrm{min}$.), $4^{\circ} \mathrm{C} 70 \%$ ethanol ( $2 \mathrm{~min}$.), $4^{\circ} \mathrm{C} 95 \%$ ethanol ( $2 \mathrm{~min}$.), $4^{\circ} \mathrm{C} 100 \%$ ethanol $(2 \mathrm{~min}),. 4^{\circ} \mathrm{C}$ $100 \%$ xylene ( $2 \mathrm{~min}.), 4^{\circ} \mathrm{C} 100 \%$ xylene ( $2 \mathrm{~min}$.), and finally RT $100 \%$ xylene $(2 \mathrm{~min}$.)). Slides were allowed to dry in a fume hood for $30 \mathrm{~min}$.

\section{Laser Pressure Catapult Micro-dissection (LPCM)}

The P.A.L.M. Microbeam ${ }^{\mathrm{TM}}$ System (Zeiss) was used to harvest cells of interest. Only the pressure catapult function was used and the laser energy setting for the pressure catapult function was minimized. The laser focus was fixed, but the objective focus was manually adjusted to ensure efficient removal of tissues of interest during LPCM. Dissected tissue was captured in opaque adhesive cap $0.5 \mathrm{~mL}$ tubes (Zeiss, part \#415101-4400-250) and stored at $-80^{\circ} \mathrm{C}$.

\section{RNA extraction and RNA cleanup}

Total RNA was extracted from LPCM harvested material using the PicoPure ${ }^{\mathrm{TM}}$ RNA isolation kit (Arcturus) with adaptations derived, in part, from documentation accompanying the opaque adhesive cap $0.5 \mathrm{~mL}$ tubes (Zeiss, part \#415101-4400-250). The changes include modification of the RNA extraction step as detailed in the Arcturus protocol (Step 1) and are as follows: We removed the adhesive cap and placed it into the tube body of a $0.5 \mathrm{~mL}$ Eppendorf Safe-Lock $^{\text {TM }}$ tube (part \# 022363719) to achieve a better tube-cap seal. $50 \mu \mathrm{L}$ of buffer $\mathrm{XB}$ was added to the tube and it was vortexed inverted for 30 seconds. The tube was then placed in a temperature equilibrated $\left(42^{\circ} \mathrm{C}\right)$ custom clamping device (not shown) to prevent leakage during the lysis step, in which the tube is inverted and incubated at $42^{\circ} \mathrm{C}$ for $30 \mathrm{~min}$. in an air incubator. At $10 \mathrm{~min}$. intervals the entire clamping device containing the tubes was vortexed inverted for 30 seconds. After the adapted lysis step we followed the PicoPure ${ }^{\mathrm{TM}}$ RNA isolation kit protocol beginning at step 2, RNA isolation. RNA was DNase treated on-column according to Appendix $\mathrm{A}$ in the PicoPure ${ }^{\text {тм }}$ RNA isolation kit protocol. Total RNA was assessed on the Agilent Bioanalyzer using the RNA 6000 Nano kit (Agilent) with the Plant Total RNA assay with specific attention to the RNA Integrity Number (RIN, scale of 1 (degraded) to 10 (intact)) [88]. An additional clean-up of the total RNA prep was required to remove what we suspected to be poly-phenolics and secondary metabolites that interfered with downstream enzymatic treatments. The RNA Clean 
and Concentrator ${ }^{\mathrm{TM}}-5$ kit (Zymo Research) was used to clean and concentrate the total RNA extracted from LPCM harvested samples following the General Procedure in protocol version 2.0.6 and RNA was eluted in $10 \mu \mathrm{L}$ RNase-free water.

\section{T7 based RNA amplification of mRNA}

The Message Amp ${ }^{\text {TM }}$ II aRNA kit (Ambion) was used to amplify the poly-A RNA contained in total RNA samples to yield amplified RNA (aRNA). The input amount was approximately $100 \mathrm{ng}$ of total RNA. The manufacturer's instructions were followed and the first round of in vitro transcription (IVT) was allowed to progress for $14 \mathrm{hrs}$. The entire aRNA sample was concentrated in a Speed-vac (Savant) to $<10$ uls then entered into second round cDNA synthesis. The second round IVT was allowed to progress for 4 hours. Total RNA was assessed on the Agilent Bioanalyzer using the RNA 6000 Nano kit (Agilent) with the mRNA assay. Typical yields after the first round of amplification were up to $100 \mathrm{ng}$ aRNA and yields after the second round of amplification ranged from 50-100 ug aRNA. High quality Arabidopsis young leaf RNA was used as a positive control and RT-PCR grade water (Ambion, included with the Message AmpII kit) was used as a negative control for amplification.

\section{Illumina paired-end library construction}

aRNA was used as input in Illumina's mRNA Seq library prep protocol (Rev D). We omitted the poly-A selection step and moved directly to "Fragment the RNA." 100 ng aRNA was fragmented and the manufacturer's instructions were followed for the rest of the library preparation with the following exceptions: 1) we size selected the adapterligated library fragments at $300 \mathrm{bp}$ rather than $200 \mathrm{bp}$ at the "Purify the cDNA Templates" step, 2) we performed a second size selection/purification step by running a gel in a similar fashion as described in the "Purify the cDNA Templates" step and excised the band at approximately $325 \mathrm{bp}$ that contained the products of library enrichment. The second size selection was done to purify the library and further constrain the fragment distribution as recommended by Illumina for paired-end mRNA Seq. The Illumina RNA Seq library was assessed on the Agilent Bioanalyzer using the DNA 1000 kit (Agilent).

\section{Sequencing, bioinformatics and phylogenetics Sequencing}

Paired-end $(83 \times 83 \mathrm{bp})$ sequencing was performed on the Illumina Genome Analyzer $2 x$ by the Genomics Core facility at the University of Virginia in Charlottesville, VA USA. Each library was sequenced in one lane.

\section{Post sequencing data processing and annotation}

Contaminating sequences were removed from the preassembled, paired-end reads by alignment to the annotated coding DNA sequences of Medicago truncatula [38] and Zea mays [38] genomes using version 1.1.0014 of Mosaik Assembler [89] with the recommended parameters ( $\mathrm{hs}=15, \mathrm{~mm}=12$, and act $=35)$. Unaligned reads were then trimmed to remove low-quality bases $(<\mathrm{Q} 20)$ from the ends using the quality trim program of CLC Assembly Cell version 3.2 (http://www.clcbio.com/index.php) requiring additionally that the remaining read fragment be at least half the original read length. Paired-end read files were reconstructed from the trimmed read fragments and orphaned single-end read fragments written to separate files using a custom script. The resultant filtered and trimmed set of reads for each library was then de novo assembled using the Inchworm component in release 03122011 of Trinity [30] with default parameters. Assemblies were filtered using version 2 of ESTScan [90] to remove sequences that had numerous frame-shift errors in coding regions, and version 4.0 of USEARCH [91] to remove similar (sub)sequences (to create non-redundant de novo assemblies). The post-processed unigenes for each build were then queried (BLASTx, 1e-5) against the 10 genomic proteomes in PlantTribes $2.0[36,37]$ and assigned to PlantTribes 2.0 Orthogroups based on the cluster containing the best BLAST hit. Expression levels for each unigene was determined by mapping reads back to de novo assemblies (each interface transcriptome as well as TrVeBC1 and TrVeBC2) and computing RPKM value with the Highthroughput Sequencing RNA-Seq Analysis program of CLC Genomics Workbench version 4.6 (parameters: mismatch cost $=2$, insertion cost $=3$, deletion cost $=3$, length fraction $=0.5$, similarity $=0.8$, $\min$ insert size $=100$, and $\max$ insert size $=250$ ).

\section{Post annotation assembly filtering}

To determine the appropriate pairwise identity (at the nucleotide level) to reference host sequences, BLASTn was used to determine the frequency of unigene pairwise identity to reference host ESTs (Zea mays and Medicago truncatula, mRNA ESTs [39]). The interface transcriptomes of $T$. versicolor grown on $Z$. mays and $M$. truncatula were BLASTed into both the $Z$. mays and $M$. truncatula EST databases. The BLAST to the non-host databases was to determine the incident nucleotide pairwise identity of unigenes to a set of host references that should not be present in the assembly. To determine if unigenes with $>95 \%$ identity to reciprocal host sequences were due to cross contamination, the distribution of unigene pairwise identity was determined for a whole-plant normalized assembly (of an non-parasitic member of the Orobanchaceae, Lindenbergia philippensis (PPGP assembly LiPhGnB1, [23]). An identity threshold of $95 \%$ was established to 
minimize host contamination while retaining parasite transcripts with incidentally high identity to the host reference sequences.

Final assemblies were filtered by BLASTn to remove unigenes with $>95 \%$ identity to host derived sequences (Zea mays and Medicago truncatula, transcripts from [38], mRNA ESTs from [39]). Remaining unigenes were then filtered by BLASTn to available PPGP transcriptome assemblies of T. versicolor [23] to select for unigenes with $>95 \%$ identity to other putative Triphysaria transcripts. Unigenes with less than $95 \%$ identity to host or T. versicolor sequences were queried against the non-redundant protein sequences database [40] using BLASTx (1e-10). Sequences were sorted by best-hit species into non-plant and plant categories. Sequences that remained unannotated and unclassified after extensive efforts detailed above were translated based on the ESTScan ORF prediction and submitted to InterProScan [41] via blast2go [92] using default parameters. These unigenes were also submitted to the OrthoMCL DB [42] database using default parameters.

The unigenes with the greatest RPKM in unique and overlapping Orthogroups, excluding those belonging to Orthogroups shared in all three transcriptomes and those unique to the above ground transcriptome, were used to query the non-redundant protein sequences database (NR) at NCBI [40] with BLASTx (1e-10) using blast2go [92]. This set of 120 unigenes was queried against a collection of 17 annotated plant genomes using BLASTn and BLASTx to determine best hits to annotated plant sequences not necessarily present in NR. The 17 genomes queried are as follows: Arabidopsis thaliana, Carica papaya, Fragaria vesca, Glycine max, Medicago truncatula, Mimulus guttatus, Oryza sativa, Phoenix dactylifera, Populus trichocarpa, Selaginella moellendorffii, Solanum lycopersicum, Solanum tuberosum, Sorghum bicolor, Thellungiella parvula, Theobroma cacao, Vitis vinifera, and. Additionally, these unigenes were annotated with InterProScan [41] via blast2go [92] with the default settings.

\section{GO Slim category analysis}

An annotated $T$. versicolor PPGP transcriptome with no tissue overlap of interface transcriptomes (TrVeBC1, [23]) was included in Orthogroup analysis to serve as a point of comparison for interface transcriptome analysis. Putative $T$. versicolor unigenes (excluding host and non-plant unigenes) were sorted based on Orthogroup assignment using Venny [93]. GO Slim annotations from unigenes present in Orthogroups were subject to a Chi-Square test using $\mathrm{R}$ [94]. Alpha was set to 0.05 .

\section{Phylogenetic analysis of TVEXPB1 and TVEXPA4}

Homologs of TvEXPB1 from Arabidopsis thaliana, Oryza sativa, Mimulus guttatus, and Selaginella moellendorfii were extracted from Phytozome [38] v7.0: gene family
\#28891348 ( $\beta$-expansins). Separately, alpha expansin homologs of TvEXPA4 were extracted from PlantTribes 2.0 [36] Orthogroup 1292. These sequences were combined with a subset of translatable sequences assembled in the PPGP project that had best BLAST hits with PlantTribes v2.0 Orthogroup 6163 ( $\beta$-expansins) and 1292 ( $\alpha$-expansins). Inferred amino acid sequences for each data set were aligned using MUSCLE [95] and the coding DNA sequences were then forced onto this alignment for phylogenetic analysis. RAxML [96] version 7.2.8 was used to estimate the maximum likelihood tree under the GTR+gamma model of molecular evolution and bootstrap support values were estimated using 100 rapid bootstrapping replicates.

\section{Verification of Expansin transcript sequence and relative transcript abundance cDNA synthesis}

cDNA was synthesized from the same amplified samples used for sequencing, plus an additional biological replicate of each, to use as a template for qRT-PCR using the iScript $^{\mathrm{TM}}$ Reverse Transcription Supermix for RT-qPCR (BioRad). The manufacturer's instructions were followed for all RNA samples as this kit utilizes both oligo-dT and random priming.

\section{RNA isolation from host roots}

RNA was isolated from host roots that were co-cultured, as described above, with $T$. versicolor. Host roots were harvested at sites adjacent to haustoria and flash frozen on liquid $\mathrm{N}_{2}$. Approximately $50 \mathrm{mg}$ of tissue was vigorously macerated in Kimble Chase glass tissue grinders (part \# KT885450-0020) in the presence of $450 \mu \mathrm{L}$ buffer $\mathrm{RLT}+\beta$-mercapto-ethanol (Qiagen) for $3 \mathrm{~min}$. The lysate was then transferred to a QiaShredder column and the RNeasy Plant Mini Kit (Qiagen) instructions were then followed with the addition of an on-column DNase treatment (Appendix D: RNeasy Mini Handbook $4^{\text {th }}$ Edition) until elution, which was done in $30 \mu \mathrm{L}$ RNase free water. Total RNA was assessed on the Agilent Bioanalyzer using the RNA 6000 Pico kit (Agilent) with the Plant Total RNA assay.

\section{Primer design and sequence verification}

Primers were designed using Geneious Pro (v5.5.4 [97]) to amplify TvEXPA4 and TvEXPB1 from aRNA based upon the unigenes that resulted from the de novo assembly and post-processing of the Illumina paired-end mRNA Seq data.

Primer sequences:

TvEXPA_F5: GCTTTTGCCTACGACCAACTTATG

TvEXPA_R3: GACAGTTTTGCCATCGCTTGTAG

TvEXPB_F1: GCCATAGTTTCAACCCGAGGAC

TvEXPB_R2: GGCTTCTTCCTGCTCTCCTTACTTG 
With these primers the putative $\alpha$ - and $\beta$-expansins transcripts were amplified and submitted for Sanger sequencing. Sequencing reads were quality trimmed manually by visually examining the electroferrograms. MUSCLE was used to align the Sanger reads with the unigenes to confirm the unigene sequence. Any remaining gaps were sequenced with the same method using primers based on the Sanger sequence verified first-round PCR products.

\section{Quantitative Real-Time Polymerase Chain Reaction}

(qRT-PCR) To verify the host-specific expression pattern of TvEXPB1 and TvEXPA4 observed in the de novo read mapping results, transcript levels of TvEXPB1, TvEXPA4, TvActin, ZmActin, and MtActin were estimated using qRT-PCR.

Primer sequences:

TvEXPA4:

For: 5'-TGGGAGGTGCTTGTGGGTAT-3';

Rev: 5'-CCGCAGGATAACCCATTGTT-3'

TvEXPB1:

For: 5'-GATGGCCTGACTGAAGTTGCA-3';

Rev: 5'-GCGGCAAATTCACCCTAAAA-3'

TvActin:

For: 5'-ACCCGATCCTTCTCACTGA-3';

Rev: 5'-CATGACAATACCAGTCGTACG-3'

ZmActinB:

For: 5'-CAATGGCACTGGAATGGT-3';

Rev: 5'-ATCTTCAGGCGAAACACG-3' [98]

MtActin:

For: 5'-ATGTTGCTATTCAGGCCG-3';

Rev: 5'-GCTCATAGTCAAGGGCAAT-3' [99]

\section{Verification of primer specificity}

To determine if the primers were specific to their intended targets, melt-curve analysis was performed for each PCR product. Primer specificity for parasite target genes was verified by submitting host RNA extracted from cocultured host roots to analysis by qRT-PCR with parasite gene primers. In both cases host actin transcripts were detected, yet primer pairs specific to the parasite genes yielded signal consistent with background. All no-template controls (NTC) showed signal consistent with background signal and all reverse transcription negative (RTN) controls showed signal consistent with background.

\section{qRT-PCR assay conditions}

The qRT-PCR reaction prepared using the KAPA ${ }^{\mathrm{TM}}$ SYBR FAST qPCR kit (KAPA Biosystems, KK4602) following the manufacturer's instructions. The reaction was run on a BioRad MyiQ (170-9770) with the following program:

$95^{\circ} \mathrm{C}$ for $8 \mathrm{~min}$. (initial melt)

$95^{\circ} \mathrm{C}$ for $0.5 \mathrm{~min}$. (cycle melt)

$60^{\circ} \mathrm{C}$ for $0.5 \mathrm{~min}$. (cycle anneal/extend)
Repeat 40 cycles

Melt Curve: $0.5^{\circ} \mathrm{C}$ increments from $95^{\circ} \mathrm{C}-25^{\circ} \mathrm{C}$.

\section{qRT-PCR data analysis}

Crossing point $(\mathrm{Ct})$ values for each of 3 technical replicates were used to calculate the average $C t$ value. The $2^{\wedge}(-\mathrm{ddCt})$ method was used to calculate the fold-change in expression in each sample relative to the control [100]. A one-tailed, two-sample $t$-test assuming unequal variance was performed using R [94]; alpha was set to 0.05 .

\section{Additional files}

\begin{abstract}
Additional file 1: Figure S1. Unigene Pairwise Nucleotide Identity Plot. Sequence identity between unigenes considered in this study and reference EST sets (PlantGDB public ESTs, http://www.plantgdb.org/) for the hosts $Z$. mays and $M$. truncatula. Triphysaria unigenes were aligned to the host reference to identify host contaminants and aligned to the reciprocal non-host reference sets to identify the incidental nucleotide pairwise identity. A whole plant normalized transcriptome assembly of Lindenbergia philippensis (a non-parasitic member of the Orobanchaceae) was used to determine the distribution of pairwise identity for a nonparasite to each host and to control for high unigene identity to host ESTs from potential cross contamination. A threshold of $95 \%$ was chosen to balance exclusion of host transcripts with retention of Triphysaria unigenes that had incident high identity to host ESTs.
\end{abstract}

Additional file 2: Figure S2. VENN diagram summary of OrthoMCL DB and InterProScan (IPS) results. ESTScan ORF predictions from unigenes in each interface transcriptome that remained unclassified after extensive BLAST-based database searching were translated and submitted to OrthoMCL DB and InterProScan. The pattern is similar between unigenes from each transcriptome indicating equivalent unigene classification for T. versicolor grown on both hosts. The number of unigenes for which an ortholog or peptide motif was identified was relatively small, indicating our unigene classification using PlantTribes 2.0 and external database queries was robust. Approximately $25 \%$ of the known orthologs identified in the OrthoMCL database from each transcriptome are shared.A majority of the unigenes remain unknown, and these include many ( $\sim 500$ in each transcriptome) that are $>300$ nucleotide bp and have read support.

Additional file 3: OrthoMCL DB and InterProScan annotation summary spreadsheet of unigenes that remained after the screen of PPGP databases, host CDNA and EST sequences, and NCBI's nonredundant protein sequences database.

Additional file 4: Figure S3. GO Slim category analysis.

Chi-Square test $(P<<0.0001)$ of GO Slim terms represented in the indicated regions of the Venn. The numbers of unigenes in each $\mathrm{GO}$ category for indicated regions are listed in the table. Cells with strongly positive residual values $(>4)$ are indicated as bold+ and strongly negative residual values $(<-4)$ are indicated as bold-. GO Slim Function $(\mathbf{A})$, Component (B) and Process $(\mathbf{C})$ category analysis for the interface transcriptome of T. versicolor grown on Z. mays. GO Slim Function (D), Component $(\mathbf{E})$ and Process $(\mathbf{F})$ category analysis for the interface transcriptome of $T$. versicolor grown on $M$. truncatula.

Additional file 5: Figure S4. Correlation of normalized read counts (RPKM) for unigenes in orthogroups shared between the interface transcriptomes and reference assembly TrVeBC1 (ppgp.huck.psu.edu). Reads from each interface transcriptome were mapped to a reference assembly (TrVeBC2, ppgp.huck.psu.edu) that included whole haustorium data from $T$. versicolor grown on $M$. truncatula. A subset of unigenes is more highly expressed in the interface transcriptome of $T$. versicolor grown on $M$. truncatula; a similar pattern is not observed for T. versicolor grown on Z. mays. This is due to a bias for Medicago grown Triphysaria unigenes in the reference dataset TrVeBC2, which was constructed with reads from Medicago grown Triphysaria. For unigenes in shared orthogroups, the RPKM values are highly 
correlated (Pearson's $R=0.81$ ) between interface transcriptomes indicating that technical and biological variation is low.

Additional file 6: Figure S5. Highly Expressed Interface Unigenes. The 20 most highly expressed (RPKM) unigenes (ID) in each indicated portion of the transcriptome Venn diagram for the interaction of T. versicolor with each host species. NR BLASTX - description, species and \%id.: the description, species of origin, and percent pairwise identify, respectively, of the best unigene alignment $(<1 \mathrm{e}-10)$ resulting from the NR database query, 17 genomes BLAST and \%id.: best hit species in a BLAST database of 17 annotated plant genomes with the percent pairwise identity in the nucleotide BLAST (N) or translated nucleotide BLAST (P). ${ }^{\top} X X X=I P S$ transmembrane prediction, ${ }^{5} X X X=I P S$ secretion signal prediction

Additional file 7: Text file (BLAST default output format) of BLAST results from the query of NCBI's non-redundant protein sequences database with $Z$. mays grown $T$. versicolor unigenes listed in Additional file 6 .

Additional file 8: Text file (BLAST default output format) of BLAST results from the query of NCBI's non-redundant protein sequences database with $M$. truncatula grown $T$. versicolor unigenes listed in Additional file 6.

Additional file 9: Results from the InterProScan analysis of $Z$. mays grown $T$. versicolor unigenes listed in Additional file 6.

Additional file 10: Results from the InterProScan analysis of $M$. truncatula grown $T$. versicolor unigenes listed in Additional file 6 .

Additional file 11: Figure S6. RaxML analysis of A: Triphysaria beta expansin gene TvEXPB1 (TrVelntZeamaGB1_772, green text), and B: alpha expansin gene TvEXPA4 (TrVelntMedtrGB1_11, green text). Bootstrap proportions are given above each node. Taxon abbreviations for A: Arabidopsis thaliana (AT), Oryza sativa (Os), Mimulus guttatus (Mg), Triphysaria versicolor (TrVe), Striga hermonthica (StHe), Phelipanche (=Orobanche) aegyptiaca (OrAe), Selaginella moellendorffii (Smoellendorffii). Taxon Abbreviations for B: Oryza sativa (Os), Sorghum bicolor (Sb), Striga hermonthica (StHe), Phelipanche (=Orobanche) aegyptiaca (OrAe),

Triphysaria versicolor (TrVe), Carica papaya (Carpa), Populus trichocarpa (Poptr), Medicago truncatula (Medtr), Vitis vinifera (Vitvi), Arabidopsis thaliana (AT), Selaginella moellendorffii (Selmo), Physcomitrella patens (Phypa).

\section{Abbreviations}

(NGS): Next Generation Sequencing; (LPCM): Laser Pressure Catapult Microdissection; (ROI): Regions of Interest; (RIN): RNA Integrity Number; (aRNA): amplified RNA; (T. versicolor): Triphysaria versicolor; (Z. mays): Zea mays; (M. truncatula): Medicago truncatula; (RPKM): Reads/kilobase/million mappable reads; (LM): Laser Micro-dissection; (PR): Pathogen resistance; (qRTPCR): Quantitative Real-Time PCR; (NCBI): National Center for Biotechnology Information; (NR): Non-redundant protein sequences database.

\section{Competing interests}

The authors declare that they have no competing interests.

\section{Authors' contributions}

Conception and design of PPGP transcriptome (JHW "CWD, MPT, JIY) and interface transcriptome study (LAH, CWD); seeds and Triphysaria growth and assays: JIY; plant cultivation and tissues: LAH; laser capture methods and sampling: LAH, CGT; RNAs, amplifications, and sequencing libraries: LAH; GRT-PCR: LAH, ZY; data analysis and presentation: LAH, JPD, EKW, NJW, ZY, NSA, CWD; Wrote manuscript: LAH and CWD with contributions from all of the authors. All authors read and approved the final manuscript.

\section{Acknowledgments}

The authors gratefully acknowledge partial funding for this project through NSF Plant Genome award DBI-0701748 to Westwood JH, dePamphilis CW, Timko MP, and Yoder JI, NSF award IOS-0821954 and IOS-1104334 to Taylor CG, McIntyre L and Koch, K, and the Intercollege Graduate Program in Plant Biology and Department of Biology of Penn State University. We thank Paula Ralph for lab assistance and Yongde Bao (University of Virginia) for Illumina sequencing. We also thank Daniel J. Cosgrove for helpful discussion and encouragement throughout the course of this research. We thank dePamphilis lab members past and present for their assistance with various aspects of this work, and two anonymous reviewers for helpful comments.

\section{Author details}

${ }^{1}$ Intercollege Graduate Program in Plant Biology, Huck Institutes of the Life Sciences, The Pennsylvania State University, University Park, Pennsylvania 16802, USA. ${ }^{2}$ Department of Biology and Institute of Molecular Evolutionary Genetics, The Pennsylvania State University, University Park, Pennsylvania 16802, USA. ${ }^{3}$ Department of Statistics and Huck Institutes of the Life Sciences, The Pennsylvania State University, University Park, Pennsylvania 16802, USA. ${ }^{4}$ Department of Plant Pathology, The Ohio State University, Ohio Agricultural Research and Development Center, Wooster, OH 44691, USA. ${ }^{5}$ Department of Plant Sciences, University of California, Davis, Davis, California 95616, USA. ${ }^{6}$ Department of Biology, University of Virginia, Charlottesville, VA 22904, USA. ${ }^{7}$ Department of Plant Pathology, Physiology and Weed Science, Virginia Polytechnic Institute and State University, Blacksburg, VA 24061, USA. ${ }^{8}$ Present address: Chicago Botanic Garden, Glencoe, IL 60022, USA.

Received: 31 August 2012 Accepted: 17 December 2012 Published: 9 January 2013

\section{References}

1. Westwood JH, Yoder Jl, Timko MP, dePamphilis CW: The evolution of parasitism in plants. Trends Plant Sci 2010, 15(4):227-235.

2. Olmstead RG, dePamphilis CW, Wolfe AD, Young ND, Elisons WJ, Reeves PA: Disintegration of the Scrophulariaceae. Am J Bot 2001, 88(2):348-361

3. Ejeta G: The Striga scourge in Africa: a growing pandemic. In Integrating New Technologies for Striga Control: Towards Ending the Witch-hunt. Edited by Gressel J. Singapore: World Scientific Publishing Co; 2007:3-16.

4. Scholes JD, Press MC: Striga infestation of cereal crops-an unsolved problem in resource limited agriculture. Curr Opin Plant Biol 2008, 11(2):180-186.

5. Parker C: Observations on the current status of Orobanche and Striga problems worldwide. Pest Manag Sci 2009, 65(5):453-459.

6. Vurro M, Bonciani B, Vannacci G: Emerging infectious diseases of crop plants in developing countries: impact on agriculture and socioeconomic consequences. Food Secur 2010, 2(2):113-132.

7. Press MC, Graves JD: Parasitic Plants. London: Chapman and Hall; 1995.

8. Tomilov A, Tomilova N, Yoder Jl: Agrobacterium tumefaciens and Agrobacterium rhizogenes transformed roots of the parasitic plant Triphysaria versicolor retain parasitic competence. Planta 2007, 225(5):1059-1071.

9. Yoder J: A species-specific recognition system directs haustorium development in the parasitic plant Triphysaria (Scrophulariaceae). Planto 1997, 202(4):407-413.

10. Westwood JH, de Pamphilis CW, Das M, Fernández-Aparicio M, Honaas LA, Timko MP, Wafula EK, Wickett NJ, Yoder Jl: The parasitic plant genome project: new tools for understanding the biology of Orobanche and Striga. Weed Sci 2012, 60(2):295-306.

11. Kuijt J: The Biology of Parasitic Flowering Plants. Berkeley: University of California Press; 1969

12. Heide-Jørgensen $\mathrm{H}$, Kuijt J: Epidermal derivatives as xylem elements and transfer cells: a study of the host-parasite interface in two species of Triphysaria (Scrophulariaceae). Protoplasma 1993, 174(3):173-183.

13. Heide-Jørgensen HS, Kuijt J: The haustorium of the root parasite Triphysaria (Scrophulariaceae), with special reference to xylem bridge ultrastructure. Am J Bot 1995, 82(6):782-797.

14. Vachev T, Ivanova D, Minkov I, Tsagris M, Gozmanova M: Trafficking of the Potato spindle tuber viroid between tomato and Orobanche ramosa. Virology 2010, 399(2):187-193.

15. Birschwilks M, Haupt S, Hofius D, Neumann S: Transfer of phloem-mobile substances from the host plants to the holoparasite Cuscuta sp. J Exp Bot 2006, 57(4):911-921.

16. Tomilov AA, Tomilova NB, Wroblewski T, Michelmore R, Yoder Jl: Trans-specific gene silencing between host and parasitic plants. Plant J 2008, 56(3):389-397.

17. Thompson JN: The Coevolutionary Process. Chicago: The University of Chicago Press; 1994. 
18. Agosta SJ, Janz N, Brooks DR: How specialists can be generalists: resolving the "parasite paradox" and implications for emerging infectious disease. Zoologia (Curitiba) 2010, 27:151-162.

19. Atsatt PR, Strong DR: Population biology of annual grassland hemiparasites .1. Host Environment. Evolution 1970, 24(2):278-291.

20. The Parasitic Plant Connection. http://www. parasiticplants.siu.edu/.

21. Schneeweiss GM: Correlated evolution of life history and host range in the nonphotosynthetic parasitic flowering plants Orobanche and Phelipanche (Orobanchaceae). J Evol Biol 2007, 20(2):471-478.

22. Thurman LD: Genecological studies in Orthocarpus subgenus Triphysaria. University of California, Berkeley: PhD Thesis. Berkeley; 1966.

23. The Parasitic Plant Genome Project. http://ppgp.huck.psu.edu/.

24. Estabrook EM, Yoder Jl: Plant-plant communications: Rhizosphere signaling between parasitic angiosperms and their hosts. Plant Physio/ 1998, 116(1):1-7.

25. Jamison DS, Yoder Jl: Heritable variation in quinone-induced haustorium development in the parasitic plant Triphysaria. Plant Physiol 2001, 125(4):1870-1879.

26. Bandaranayake PCG, Filappova T, Tomilov A, Tomilova NB, Jamison-McClung $D$, Ngo Q, Inoue K, Yoder Jl: A single-electron reducing quinone oxidoreductase is necessary to induce haustorium development in the root parasitic plant Triphysaria. Plant Cell 2010, 22(4):1404-1419.

27. Offler CE, McCurdy DW, Patrick JW, Talbot MJ: Transfer cells: Cells specialized for a special purpose. Annu Rev Plant Biol 2003, 54:431-454

28. Wall PK, Leebens-Mack J, Chanderbali AS, Barakat A, Wolcott E, Liang H, Landherr L, Tomsho LP, Hu Y, Carlson JE, et al: Comparison of next generation sequencing technologies for transcriptome characterization. BMC Genom 2009, 10(1):347.

29. Strickler SR, Bombarely A, Mueller LA: Designing a transcriptome next-generation sequencing project for a nonmodel plant species. Am J Bot 2012, 99(2):257-266.

30. Grabherr MG, Haas BJ, Yassour M, Levin JZ, Thompson DA, Amit I, Adiconis $X$, Fan L, Raychowdhury R, Zeng Q, et al: Full-length transcriptome assembly from RNA-Seq data without a reference genome. Nat Biotech 2011, 29(7):644-652.

31. O'Malley RC, Lynn DG: Expansin message regulation in parasitic angiosperms: marking time in development. Plant Cell 2000, 12(8):1455-1465.

32. Wrobel RL, Yoder Jl: Differential RNA expression of alpha-expansin gene family members in the parasitic angiosperm Triphysaria versicolor (Scrophulariaceae). Gene 2001, 266(1-2):85-93.

33. Kenzelmann M, Klaren R, Hergenhahn M, Bonrouhi M, Grone HJ, Schmid W, Schutz G: High-accuracy amplification of nanogram total RNA amounts for gene profiling. Genomics 2004, 83(4):550-558.

34. Matas AJ, Yeats TH, Buda GJ, Zheng Y, Chatterjee S, Tohge T, Ponnala L, Adato A, Aharoni A, Stark R, et al: Tissue- and cell-type specific transcriptome profiling of expanding tomato fruit provides insights into metabolic and regulatory specialization and cuticle formation. Plant Cell 2011, 23(11):3893-3910.

35. Wall PK, Leebens Mack J, Muller KF, Field D, Altman NS, dePamphilis CW: PlantTribes: a gene and gene family resource for comparative genomics in plants. Nucleic Acids Res 2008, 36:D970-D976.

36. The Floral Genome Project. http://fgp.bio.psu.edu.

37. Wickett NJ, Honaas LA, Wafula EK, Das M, Huang K, Wu B, Landherr L, Timko MP, Yoder J, Westwood JH, et al: Transcriptomes of the parasitic plant family Orobanchaceae reveal surprising conservation of chlorophyll synthesis. Curr Biol 2011, 21:2098-2104.

38. Phytozome. http://www.phytozome.net.

39. PlantGBD. http://www.plantgdb.org.

40. National Center for Biotechnology Information. http://www.ncbi.nlm.nih.gov.

41. European Bioinformatics Institute. http://www.ebi.ac.uk/.

42. OrthoMCL DB. http://www.orthomcl.org/.

43. Sampedro J, Cosgrove DJ: The expansin superfamily. Genome Biol 2005, 6(12):242.

44. Brandt SP: Microgenomics: Gene expression analysis at the tissue-specific and single-cell levels. J Exp Bot 2005, 56(412):495-505.

45. Day RC, Grossniklaus U, Macknight RC: Be more specific! Laser-assisted microdissection of plant cells. Trends Plant Sci 2005, 10(8):397-406.

46. Nelson T, Gandotra N, Tausta SL: Plant cell types: Reporting and sampling with new technologies. Curr Opin Plant Biol 2008, 11(5):567-573.

47. Gomez SK, Harrison MJ: Laser microdissection and its application to analyze gene expression in arbuscular mycorrhizal symbiosis. Pest Manag Sci 2009, 65(5):504-511.
48. Woll K: Isolation, characterization, and pericycle-specific transcriptome analyses of the novel maize lateral and seminal root initiation mutant rum1. Plant Physiol 2005, 139(3):1255-1267.

49. Ithal N, Recknor J, Nettleton D, Hearne L, Maier T, Baum TJ, Mitchum MG Parallel genome-wide expression profiling of host and pathogen during soybean cyst nematode infection of soybean. MPMI 2007, 20(3):293-305.

50. Gomez SK, Javot H, Deewatthanawong P, Torres-Jerez I, Tang Y, Blancaflor EB, Udvardi MK, Harrison MJ: Medicago truncatula and Glomus intraradices gene expression in cortical cells harboring arbuscules in the arbuscular mycorrhizal symbiosis. BMC Plant Biol 2009, 9(1):10.

51. Klink VP, Hosseini P, Matsye P, Alkharouf NW, Matthews BF: A gene expression analysis of syncytia laser microdissected from the roots of the Glycine max (soybean) genotype PI 548402 (Peking) undergoing a resistant reaction after infection by Heterodera glycines (soybean cyst nematode). Plant Mol Biol 2009, 71(6):525-567.

52. Matas AJ, Agustí J, Tadeo FR, Talón M, Rose JKC: Tissue-specific transcriptome profiling of the citrus fruit epidermis and subepidermis using laser capture microdissection. J Exp Bot 2010, 61(12):3321-3330.

53. Pillitteri $L$, Peterson KM, Horst RJ, Torii KU: Molecular profiling of stomatal meristemoids reveals new component of asymmetric cell division and commonalities among stem cell populations in Arabidopsis. Plant Cell 2011, 23(9):3260-3275.

54. Emrich SJ, Barbazuk WB, Li L, Schnable PS: Gene discovery and annotation using LCM-454 transcriptome sequencing. Genome Res 2007, 17(1):69-73.

55. Ohtsu K, Smith MB, Emrich SJ, Borsuk LA, Zhou RL, Chen TL, Zhang XL, Timmermans MCP, Beck J, Buckner B, et al: Global gene expression analysis of the shoot apical meristem of maize (Zea mays L.). Plant J 2007, 52(3):391-404.

56. Li PH, Ponnala L, Gandotra N, Wang L, Si YQ, Tausta SL, Kebrom TH, Provart $N$, Patel R, Myers CR, et al: The developmental dynamics of the maize leaf transcriptome. Nat Genet 2010, 42(12):1060-1067.

57. Feldman AL, Costouros NG, Wang E, Qian M, Marincola FM, Alexander HR, Libutti SK: Advantages of mRNA amplification for microarray analysis. Biotechniques 2002, 33(4):906

58. Polacek DC, Passerini AG, Shi C, Francesco NM, Manduchi E, Grant GR, Powell S, Bischof H, Winkler H, Stoeckert CJ, et al: Fidelity and enhanced sensitivity of differential transcription profiles following linear amplification of nanogram amounts of endothelial mRNA. Physiol Genomics 2003, 13(2):147-156.

59. Li Y, Li T, Liu SZ, Qiu MY, Han ZY, Jiang ZL, Li RY, Ying K, Xie Y, Mao YM: Systematic comparison of the fidelity of aRNA, mRNA and T-RNA on gene expression profiling using cDNA microarray. J Biotech 2004, 107(1):19-28.

60. King C, Guo N, Frampton GM, Gerry NP, Lenburg ME, Rosenberg CL: Reliability and reproducibility of gene expression measurements using amplified RNA from laser-microdissected primary breast tissue with oligonucleotide arrays. J Mol Diagnos 2005, 7(1):57.

61. Day RC, McNoe LA, Macknight RC: Transcript analysis of laser microdissected plant cells. Physiol Plantarum 2007, 129(2):267-282.

62. Goldsworthy SM, Stockton PS, Trempus CS, Foley JF, Maronpot RR: Effects of fixation on RNA extraction and amplification from laser capture microdissected tissue. Mol Carcino 1999, 25(2):86-91.

63. Gillespie JW, Best CJM, Bichsel VE, Cole KA, Greenhut SF, Hewitt SM, Ahram M, Gathright YB, Merino MJ, Strausberg RL, et al: Evaluation of nonformalin tissue fixation for molecular profiling studies. Am J Path 2002, 160(2):449-457.

64. Kerk NM: Laser capture microdissection of cells from plant tissues. Plant Physiol 2003, 132(1):27-35.

65. Nakazono M: Laser-capture microdissection, a tool for the global analysis of gene expression in specific plant cell types: identification of genes expressed differentially in epidermal cells or vascular tissues of maize. Plant Cell 2003, 15(3):583-596.

66. Rispail N, Dita MA, Gonzalez-Verdejo C, Perez-de-Luque A, Castillejo MA, Prats E, Roman B, Jorrin J, Rubiales D: Plant resistance to parasitic plants: molecular approaches to an old foe. New Phytol 2007, 173(4):703-711.

67. Tomilov A, Tomilova N, Shin DH, Jamison D, Torres J, Reagan R, Horning T, Truong R, Nava A, Nava A, et al: Chemical signaling between plants: mechanistic similarities between phytotoxic allelopathy and host recognition by parasitic plants. In Chemical Communication: From Gene to Ecosystem. Edited by Dicke M. Wageningen: Frontis; 2006:55-69.

68. Cosgrove DJ: Growth of the plant cell wall. Nat Rev Mol Cell Biol 2005, 6(11):850-861. 
69. Albrecht U, Bowman KD: Gene expression in Citrus sinensis (L.) Osbeck following infection with the bacterial pathogen Candidatus liberibacter asiaticus causing Huanglongbing in Florida. Plant Sci 2008, 175(3):291-306.

70. Ding XH, Cao YL, Huang LL, Zhao J, Xu CG, Li XH, Wang SP: Activation of the indole-3-acetic acid-amido synthetase $\mathrm{GH} 3-8$ suppresses expansin expression and promotes salicylate- and jasmonate-independent basal immunity in rice. Plant Cell 2008, 20(1):228-240.

71. Gal TZ, Aussenberg ER, Burdman S, Kapulnik Y, Koltai H: Expression of a plant expansin is involved in the establishment of root knot nematode parasitism in tomato. Planta 2006, 224(1):155-162.

72. Kikuchi T, Aikawa T, Kosaka H, Pritchard L, Ogura N, Jones JT: Expressed sequence tag (EST) analysis of the pine wood nematode Bursaphelenchus xylophilus and B-mucronatus. Mol Biochem Parasitol 2007, 155(1):9-17.

73. Li ZC, Durachko DM, Cosgrove DJ: An oat coleoptile wall protein that induces wall extension in-vitro and that is antigenically related to a similar protein from cucumber hypocotyls. Planta 1993, 191(3):349-356.

74. Cho H-T, Kende H: Expansins in deepwater rice internodes. Plant Physiol 1997, 113(4):1137-1143.

75. Cosgrove DJ, Bedinger P, Durachko DM: Group I allergens of grass pollen as cell wall-loosening agents. P Natl Acad Sci USA 1997, 94(12):6559.

76. McQueenmason SJ, Cosgrove DJ: Disruption of hydrogen bonding between plant cell wall polymers by proteins that induce wall extension. P Natl Acad Sci USA 1994, 91:6574-6578.

77. McQueenmason SJ, Cosgrove DJ: Expansin mode of action on cell-walls - Analysis of wall hydrolysis, stress-relaxation, and binding. Plant Physiol 1995, 107(1):87-100.

78. Li Y, Jones L, McQueen-Mason S: Expansins and cell growth. Curr Opin Plant Biol 2003, 6(6):603-610.

79. Cosgrove DJ: Loosening of plant cell walls by expansins. Nature 2000, 407(6802):321-326.

80. Kapu NUS, Cosgrove DJ: Changes in growth and cell wall extensibility of maize silks following pollination. J Exp Bot 2010, 61(14):4097-4107.

81. Tabuchi A, Li LC, Cosgrove DJ: Matrix solubilization and cell wall weakening by beta-expansin (group-1 allergen) from maize pollen. Plant J 2011, 68(3):546-559.

82. Yennawar NH, Li L-C, Dudzinski DM, Tabuchi A, Cosgrove DJ: Crystal

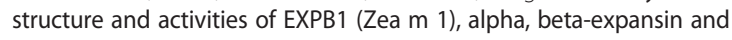
group-1 pollen allergen from maize. $P$ Natl Acad Sci USA 2006, 103(40):14664-14671.

83. Vial L, Chapalain A, Groleau MC, Deziel E: The various lifestyles of the Burkholderia cepacia complex species: a tribute to adaptation. Environ Microbiol 2011, 13(1):1-12.

84. Atsatt PR: Parasitic flowering plants: How did they evolve? Am Nat 1973 107(956):502-510.

85. Chuang TI, Heckard LR: Generic realignment and synopsis of subtribe Castillejinae (Scrophulariaceae-tribe Pediculareae). Syst Bot 1991, 16(4):644-666.

86. Fernandez-Aparicio M, Rubiales D, Bandaranayake P, Yoder J, Westwood J: Transformation and regeneration of the holoparasitic plant Phelipanche aegyptiaca. Plant Methods 2011, 7(1):36.

87. Torres MJ, Tomilov AA, Tomilova N, Reagan RL, Yoder Jl: Pscroph, a parasitic plant EST database enriched for parasite associated transcripts. BMC Plant Biol 2005, 5:24

88. Schroeder A, Mueller O, Stocker S, Salowsky R, Leiber M, Gassmann M, Lightfoot S, Menzel W, Granzow M, Ragg T: The RIN: an RNA integrity number for assigning integrity values to RNA measurements. BMC Mol Biol 2006, 7:3

89. The MarthLab. http://bioinformatics.bc.edu/marthlab/Main_Page.

90. Iseli C, Jongeneel CV, Bucher P: ESTScan: a program for detecting, evaluating, and reconstructing potential coding regions in EST sequences. In Proc Int Conf Intell Syst Mol Biol.; 1999:138-148.

91. Edgar RC: Search and clustering orders of magnitude faster than BLAST. Bioinformatics 2010, 26(19):2460-2461.

92. blast2go. http://www.blast2go.com/

93. Venny. http://bioinfogp.cnb.csic.es/tools/venny/index.htm.

94. The R Project for Statistical Computing. http://www.r-project.org/.

95. Edgar RC: MUSCLE: multiple sequence alignment with high accuracy and high throughput. Nucleic Acids Res 2004, 32:1792-1797.
96. Stamatakis A: RAxML-VI-HPC: Maximum Likelihood-based Phylogenetic Analyses with Thousands of Taxa and Mixed Models. Bioinformatics 2006, 22:2688-2690.

97. Geneious. http://www.geneious.com/.

98. Xing GF, Guo GG, Yao YY, Peng HR, Sun QX, Ni ZF: Identification and characterization of a novel hybrid upregulated long non-protein coding RNA in maize seedling roots. Plant Sci 2010, 179(4):356-363.

99. Jones KM, Sharopova N, Lohar DP, Zhang JQ, VandenBosch KA, Walker GC Differential response of the plant Medicago truncatula to its symbiont Sinorhizobium meliloti or an exopolysaccharide-deficient mutant. P Natl Acad Sci USA 2008, 105(2):704-709.

100. Pfaffl MW: A new mathematical model for relative quantification in real-timeRT-PCR. Nucleic Acids Res 2001, 29(9):e45.

doi:10.1186/1471-2229-13-9

Cite this article as: Honaas et al:: Functional genomics of a generalist parasitic plant: Laser microdissection of host-parasite interface reveals host-specific patterns of parasite gene expression. BMC Plant Biology 2013 13:9.

\section{Submit your next manuscript to BioMed Central and take full advantage of:}

- Convenient online submission

- Thorough peer review

- No space constraints or color figure charges

- Immediate publication on acceptance

- Inclusion in PubMed, CAS, Scopus and Google Scholar

- Research which is freely available for redistribution 